\title{
AGK Cutting Rules and Multiple Scattering in Hadronic Collisions
}

\author{
J.Bartels ${ }^{a}$, M.Salvadore ${ }^{a, b}$, and G.P.Vacca ${ }^{b}$ \\ ${ }^{a}$ II Inst. f. Theor. Physik, Univ. of Hamburg, Germany and \\ ${ }^{b}$ Dipartimento di Fisica and INFN, Univ. of Bologna, Italy
}

\begin{abstract}
:
We discuss the AGK rules for the exchange of an arbitrary number of reggeized gluons in perturbative QCD in the high energy limit. Results include the cancellation of corrections to single jet and double jet inclusive cross sections, both for hard and soft rescattering contributions.
\end{abstract}

\section{Introduction}

More than 30 years ago, Abramovsky, Gribov, and Kancheli in their pioneering paper [1] have pointed out that, for high energy hadron hadron scattering, the multiple exchange of Pomerons with intercept at or close to unity leads to observable effects in multiparticle final states. As an example, double Pomeron exchange predicts fluctuations of the rapidity densities of produced particles; furthermore, in double inclusive particle production multiPomeron exchange leads to long range rapidity correlations. All these results suggests that, at very high energies, hadron hadron scattering might exhibit some sort of critical behavior. As to the theoretical basis of these predictions, only fairly general properties of Regge theory have been used, especially features of particle-reggeon vertices which are quite independent of any special underlying quantum field theory. In particular, no reference has been made to QCD.

In recent years, the investigation of the small- $x$ region of hard scattering processes has lead to interest in the BFKL Pomeron [2] which, at short distances, describes the exchange of vacuum quantum numbers at high energies. In connection with saturation and the color glass condensate, both in DIS at HERA and in heavy ion collisions at RHIC also the multiple exchange of BFKL Pomerons is being addressed; a convenient framework for this is the nonlinear Balitsky-Kovchegov (BK) equation. At hadron colliders, there is more and more evidence [3] that at high energies multiple interactions of partons cannot be neglected. All this naturally suggests to perform the AGK analysis in the framework of perturbative QCD where the Pomeron is described by the sum of BFKL ladder diagrams. In particular, it will be of interest to understand how the presence of multiple scattering affects, for example, the multiplicity of jets in the final state or the rapidity correlation between two jets. A special motivation for investigating multiple scattering comes from the interest in saturation: most direct signals of the existence of saturation are expected to be seen in specific features of final states. Use of the AGK rules in pQCD has already been made in several papers $[4,5]$.

The theoretical basis for studying these rules in perturbative QCD has been laid down in [6]. A clean theoretical environment for studying high energy scattering processes in pQCD is provided by virtual photons where the mass of the photon defines the momentum scale of the QCD coupling. The coupling of two BFKL Pomerons to a virtual photon has been 
analysed in [7]; a generalization to three BFKL ladders has been investigated in [8]. It is the structure of these couplings of BFKL Pomerons to the external photon which provides the basis for performing the AGK analysis in pQCD. The most interesting applications of the pQCD AGK-analysis include deep inelastic electron proton scattering or multijet production in $p p$ collisions: in both cases one needs multi-gluon couplings to the proton. In the absence of any first-principle calculation, these couplings have to be modelled, similarly to the initial conditions of the parton densities. The perturbative analysis of the coupling of two (and more) BFKL Pomerons to virtual photons suggests that these couplings are not arbitrary: they obey certain symmetry requirements, which can be expected to be valid also beyond perturbation theory. We therefore believe that our understanding of the pQCD couplings will help to model, for example in proton proton scattering, the coupling of $n$ gluons to the proton.

In this paper we therefore begin with a brief review of the main results of [6], and we point out which features of the couplings are crucial for obtaining the AGK results ${ }^{1}$. We then propose a generalization to multiple BFKL exchange in hadron-hadron scattering. Starting from these couplings, we then re-derive, for illustration, the counting rules presented in the original AGK paper for an arbitrary number of exchanged gluons, and we apply these rules to single and double inclusive jet production cross sections. The comparison with the original AGK paper allows us to include into our analysis also soft rescattering corrections. As a specific example, we consider the double BFKL correction to the Mueller-Navelet jet cross section formula [10].

The paper is organized as follows. In section 2 we briefly review the logic behind the AGK analysis, and we summarize the results of [6]. This leads us to define a framework for studying multiple BFKL exchanges in hadron-hadron scattering. In section 3 we discuss the counting rules for inclusive cross sections, and in sections 4 and 5 we turn to single jet and double jet inclusive cross sections. A few details are put into an appendix.

\section{Reggeon unitarity, energy cuts à la AGK, and particle-Pomeron couplings in $\gamma^{*} \gamma^{*}$ scattering}

\subsection{The nonperturbative AGK rules}

In this section we briefly review the AGK strategy and its application to pQCD. We will conclude that the central task is the derivation and the study of the coupling of four (or more) reggeized gluons to virtual photons.

The original AGK paper starts from a multi-Regge pole contribution to the elastic scattering amplitude (Fig.1), written as a Sommerfeld-Watson representation:

$$
\mathcal{T}_{A B}(s, t)=\int \frac{d \omega}{2 i} \xi(\omega) s^{1+\omega} \mathcal{F}(\omega, t)
$$

with $\omega=J-1$,

$$
\xi(\omega)=\frac{\tau-e^{-i \pi \omega}}{\sin \pi \omega}=i \frac{e^{-i \frac{\pi}{2}\left(\omega+\frac{1-\tau}{2}\right)}}{\cos \left[\frac{\pi}{2}\left(\omega+\frac{1-\tau}{2}\right)\right]}=i-\left[\tan \frac{\pi}{2}\left(\omega+\frac{1-\tau}{2}\right)\right],
$$

and $\tau= \pm 1$ being the signature. The (real-valued) partial wave $\mathcal{F}(\omega, t)$ has singularities in the complex $\omega$-plane, and the multi-Regge exchange corresponds to a particular branch cut.

\footnotetext{
${ }^{1}$ The issue of AGK rules in connection with multiparticle interaction has already been addressed in a different way in [9] without investigation of the cancellations involved.
} 


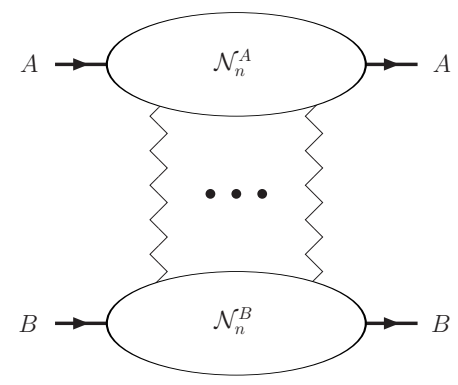

Figure 1: Graphical representation of a multi-Regge poles contribution to the elastic scattering amplitude. The zigzag lines represent pomerons.

There is a general formula for the discontinuity across this cut [11] (Fig.2a):

$$
\begin{gathered}
\operatorname{disc}_{\omega}^{(n)}[\mathcal{F}(\omega, t)]=2 \pi i \int \frac{d \Omega_{n}}{n !} \gamma_{\left\{\beta_{j}\right\}} \mathcal{N}_{n}^{A}\left(\left\{\boldsymbol{k}_{j}\right\} ; \omega\right) \mathcal{N}_{n}^{B}\left(\left\{\boldsymbol{k}_{j}\right\} ; \omega\right) \delta\left(\omega-\Sigma_{j} \beta_{j}\right) \\
d \Omega_{n}=(2 \pi)^{2} \delta^{2}\left(\boldsymbol{q}-\Sigma_{j} \boldsymbol{k}_{j}\right) \prod_{j=1}^{n} \frac{d^{2} \boldsymbol{k}_{j}}{(2 \pi)^{2}}
\end{gathered}
$$

where $\boldsymbol{k}_{j}(j=1, \ldots, n)$ denotes the transverse momentum of the $j$-th Regge pole, $\boldsymbol{q}$ is the sum over all transverse momenta with $\boldsymbol{q}^{2}=-t$, and $\alpha\left(-\boldsymbol{k}_{j}^{2}\right)=\alpha_{j}=1+\beta_{j}$ is the Regge pole trajectory function. The factor which determines the overall sign has the form

$$
\gamma_{\left\{\beta_{j}\right\}}=\Im\left[-i \Pi_{j}\left(i \xi_{j}\right)\right]=(-1)^{n-1} \frac{\cos \left[\frac{\pi}{2} \sum_{j}\left(\beta_{j}+\frac{1-\tau_{j}}{2}\right)\right]}{\prod_{j} \cos \left[\frac{\pi}{2}\left(\beta_{j}+\frac{1-\tau_{j}}{2}\right)\right]}
$$

As an example, the contribution of two even-signature Regge poles (Pomerons) with intercept close to unity is negative compared to the single pole contribution. Equation (3) is a 'reggeon unitarity equation': it describes the contribution of the $n$-reggeon $t$-channel state to the discontinuity in angular momentum of the partial wave $\mathcal{F}$ (Fig.2). In the same way as in a usual unitarity integral particles in the intermediate state are to be taken on mass shell, in the reggeon unitarity integral the reggeons of the intermediate state are on shell in reggeon energy: as indicated in Fig.2c, the Regge pole is a bound state of (at least) two particles, and the complex angular momentum of the two particles is put equal to the trajectory function of the Regge pole. The formula (3) contains the coupling of $n$ Regge poles to the external particles, denoted by $\mathcal{N}_{n}\left(\boldsymbol{k}_{j}, \omega\right)$. In general, they are functions of $\omega$ and contain, for example poles and cuts due to the exchange of Regge poles. This includes, in particular, the possibility that the reggeons $i$ and $j$ form a composite state. Depending on the structure of the $\mathcal{N}_{n}$, it may be necessary to replace, in (3), the $\omega$-dependent $\delta$-function by $\prod_{1}^{n}\left(\int d \omega_{j} \delta\left(\omega_{j}-\beta_{j}\right)\right) \delta\left(\omega-\sum_{j} \omega_{j}\right)$ : in this case, the vertex function $\mathcal{N}_{n}$ may depend not only upon the total angular momentum $\omega$, but also upon the $\omega_{j}$. An example will be given futher below. For these reasons, the vertex functions $\mathcal{N}_{n}$ are more general than the impact factors.

For later considerations it will be useful to say a few more words about the origin of this formula. Following the idea of Gribov, Pomeranchuk and Ter-Martirosian [12] one starts, in the simplest case, from the four particle intermediate state in the $t$-channel unitarity equation in the physical region of the process $A+\bar{A} \rightarrow B+\bar{B}$ (Fig.2b). Above the 4-particle state, the $2 \rightarrow 4$ amplitude for the process $A+\bar{A} \rightarrow 1+2+3+4$ appears. This scattering 


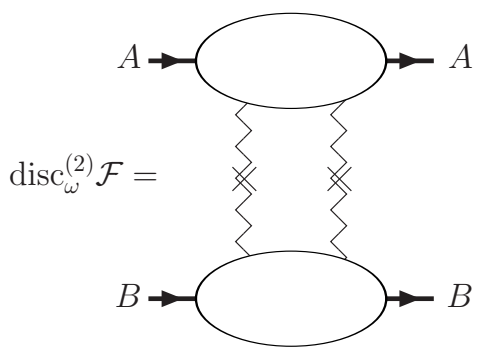

$a$

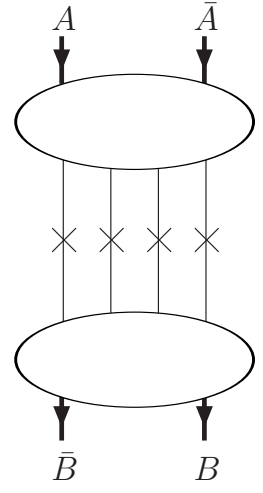

$b$

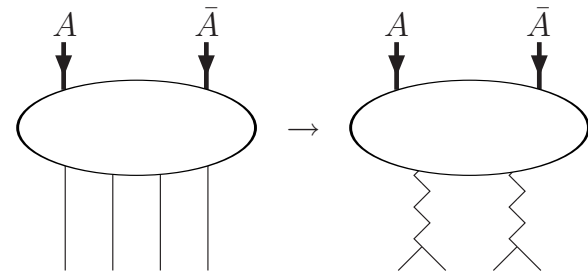

$\begin{array}{llll}1 & 2 & 3 & 4\end{array}$

$1 \quad 2 \quad 3 \quad 4$

$c$

Figure 2: Representation of the $t$-channel content of the elastic amplitude: a) discontinuity across the 2-poles cut in the $\omega$-plane, b) computation of the coupling $2 \rightarrow 4$ in the $t$-channel physical region and c) isolation of the Regge poles in the (12) and (34) channels. The crosses in a), b) indicate that the lines are on shell in angular momentum and 4-momentum, resp.

amplitude (and the corresponding one below the 4-particle state) is expanded in terms of partial waves, inserted into the $t$-channel unitarity equation. Defining analytic continuations in angular momentum and helicity variables, using Sommerfeld-Watson transformations, and performing parts of the $t$-channel unitarity phase space integrals, one moves from the physical $t$-channel region to negative $t$-values. Retaining Regge poles in the (12) and (34) channels (illustrated in Fig.2c), one finally arrives at the discontinuity of the $t$-channel partial wave across the two-reggeon cut, given by (3). This form of $t$-channel unitarity is called 'reggeon unitarity': in the Regge description of high energy scattering processes the partial waves satisfy reggeon unitarity relations, and the single pole, double, triple... exchanges can be isolated by computing discontinuities across the corresponding cuts in the angular momentum plane. The analytic form of the discontinuity equation given in (3) is universal, whereas the reggeon-particle couplings entering the equation, $\mathcal{N}_{n}$, have to be computed from the underlying theory.

The central goal of the AGK analysis is the decomposition of the n-reggeon exchange contribution in terms of $s$-channel intermediate states. To be precise, one is interested in the total cross section, i.e. in the absorptive part or, equivalently, in the discontinuity w.r.t. energy of the scattering amplitude (1). It is quite obvious that the absorptive part of the amplitude (1) will consist of several different contributions: each piece belongs to an energy cut line across Fig.1, and there are several different ways of drawing such energy-cutting lines. Each of them belongs to a particular set of $s$-channel intermediate states. For example, a cutting line between reggeons (Fig.3a) belongs to double diffractive production on both sides of the cut: there is a rapidity gap between what is inside the upper blob and the lower blob. When relating this contribution with the full diagram in Fig.1 one requires a 'cut version' of the reggeon particle couplings $\mathcal{N}_{n}$. Similarly, the cut through a reggeon (Fig.3b) corresponds to a so-called multiperipheral intermediate state, and another cut version of $\mathcal{N}_{n}$ appears. The basis of the AGK analysis is the observation that, under very general assumptions for the underlying dynamical theory, the couplings $\mathcal{N}_{n}$ are fully symmetric under the exchange of reggeons, and all their cut versions are identical. This property then allows to find simple relations between the different cut contributions, and to derive a set of counting rules. 

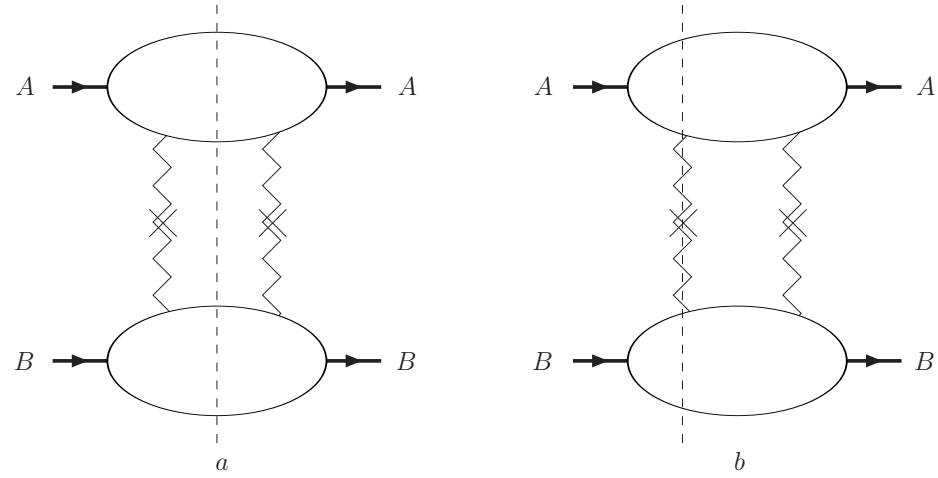

Figure 3: Some examples of the different $s$-channel cuts of the 2-pomeron contribution: a) diffractive and b) multiperipheral intermidiate states.

\subsection{The $\mathrm{pQCD}$ case}

From this brief review it follows that the central task of performing the AGK analysis in pQCD requires the computation and study of the coupling functions $\mathcal{N}_{n}$. The simplest task is the study of the two-Pomeron exchange. Since the BFKL Pomeron is a composite state of two reggeized gluons, we have to start from the exchange of four reggeized gluons (Fig.4). The blobs above and below denote the couplings $\mathcal{N}$, and they will be discussed below. They have a nontrivial content of reggeized gluons, and, in particular, any pair of gluons $1,2, \ldots, 4$ can come from a composite BFKL state contained in $\mathcal{N}$. This diagram has to be compared with Fig.1: the elementary Regge poles in pQCD are the reggeized gluons, and the Pomeron appears as a composite state. Rather than going in all detail through the chain of arguments

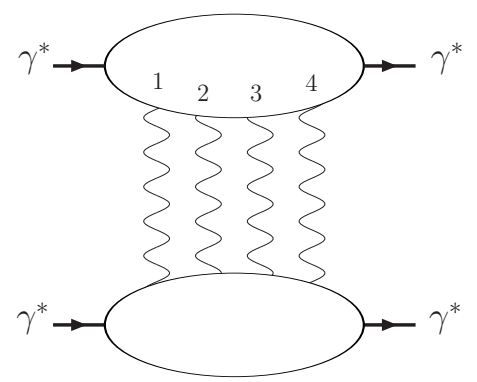

Figure 4: Exchange of four reggeized gluons in pQCD. The wavy lines represent reggeized gluons. The blobs above and below denote the functions $\mathcal{N}_{4}\left(\left\{\boldsymbol{k}_{j}\right\}, \omega\right)$, computed in pQCD.

described before (which would lead us to the study of a $t$-channel $2 \rightarrow 8$ process!), we mention a 'shortcut' path which takes us, in an easier way, to the desired coupling of four reggeized gluons to external particles. It is based upon the observation that the same coupling which appears in the reggeon unitarity equation (3) for the discontinuity across the four reggeon cut is also contained in the diffractive cross section formula for low and high-mass diffraction (triple Regge limit): the process (Fig.5) $\gamma^{*}+C \rightarrow X+C$ where $X$ sums over low and high mass diffractive states (with squared mass $M_{X}^{2}$ ) of the incoming projectile $\gamma^{*}$. In the limit of large $M_{X}$ and large energy $s$ with the restriction $M_{X}^{2} \ll s$, this process is dominated by Pomeron exchanges in the lower $t$ channels which in pQCD are BFKL ladders. By cutting these $t$-channels (more precisely: by taking cuts across the angular momentum variables in the lower $t$-channels), we arrive at the coupling of four reggeized gluons to the external particle 

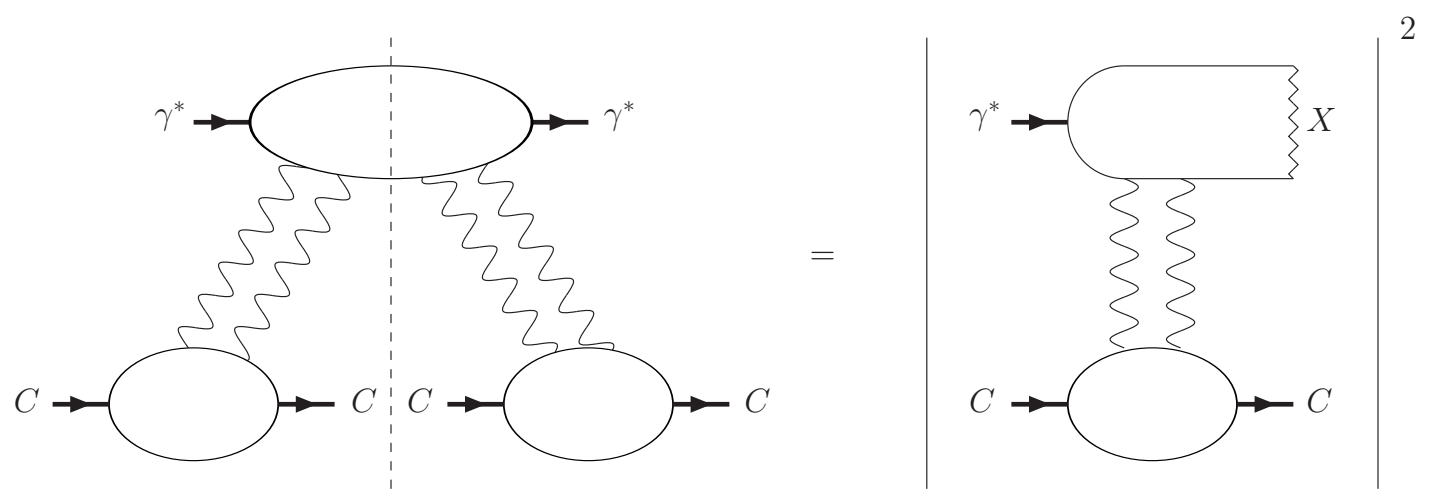

Figure 5: Six point amplitude for the computation of the high-mass diffraction cross section in the triple Regge limit.

$\gamma^{*}$. This is the method which was used in [7] for deriving the coupling of four reggeized gluons to the virtual photon, named $\mathcal{D}_{4}$. It is defined to contain the energy denominator $1 /\left(\omega-\sum_{j=1}^{4}\right)$ for the four reggeized gluons; its amputated counterpart, $C_{4}=D_{4}\left(\omega-\sum_{j=1}^{4}\right)$ will lead to a pQCD model for the coupling function $\mathcal{N}_{4}$. The study of $D_{4}$, therefore, provides the starting point for the AGK analysis in pQCD. In the following we will discuss the function $D_{4}$, keeping in mind that at the end we have to multiply by $\left(\omega-\sum_{j=1}^{4}\right)$. Before inserting this coupling into the discontinuity formula, we have to address the complications arising from the color degree of freedom and from the reggeization of the gluon.

Let us recapitulate the main properties of $\mathcal{D}_{4}$. From the $t$-channel point of view it is natural to demand that the reggeon-particle coupling that enters the reggeon unitarity equation satisfies certain symmetry requirements. In the simplest case, it should be symmetric under the exchange of the reggeons, i.e. their momenta and color labels. On the other hand, we know from the BFKL equation that there are two different $t$-channel states. The Pomeron state which belongs to the color singlet is completely symmetric; on the other hand, when the BFKL amplitude is projected onto the antisymmetric color octet, it satifies the bootstrap equation, and the two gluons 'collapse' into one single gluon. It is therefore not unexpected that also the four reggeon amplitude $\mathcal{D}_{4}$ contains antisymmetric configurations which satisfy bootstrap equations. One of the main results of the analysis of [7] is the complete decomposition of $\mathcal{D}_{4}$ into two pieces:

$$
\mathcal{D}_{4}=\mathcal{D}_{4}^{I}+\mathcal{D}_{4}^{R}
$$

Here the first term, $\mathcal{D}_{4}^{I}$, is completely symmetric under the exchange of any two gluons, whereas the second one, $\mathcal{D}_{4}^{R}$, is a sum of antisymmetric terms which, as a result of bootstrap properties, can be expressed in terms of two-gluon amplitudes, $\mathcal{D}_{2}$. In a graphical way, $\mathcal{D}_{4}^{R}$ is illustrated in Fig.6: after making use of the bootstrap properties, $\mathcal{D}_{4}^{R}$ is reduced to a sum of $\mathcal{D}_{2}$ functions. Under the exchange of the two reggeized gluons, $\mathcal{D}_{2}$ is symmetric. It is only after this decomposition has been performed, and we have arrived at reggeon particle couplings with 'good' properties, that we can start with the AGK analysis.

The construction of $\mathcal{D}_{4}^{I}$ implies that the same function appears when, in the AGK analysis, one computes different energy discontinuities of $\gamma^{*}-\gamma^{*}$ scattering, no matter where the cutting line runs, between gluon ' 1 ' and '2', or between ' 2 ' and ' 3 ', or between ' 3 ' and ' 4 '. Since in pQCD the Pomeron is a bound state of two gluons, the two-Pomeron state is formed, e.g., by the pairs (12) and (34). Thus cuts through the Pomeron or between two Pomerons all lead to the same two-Pomeron particle vertex. This means that $\mathcal{D}_{4}^{I}$ satifies all the requirements 


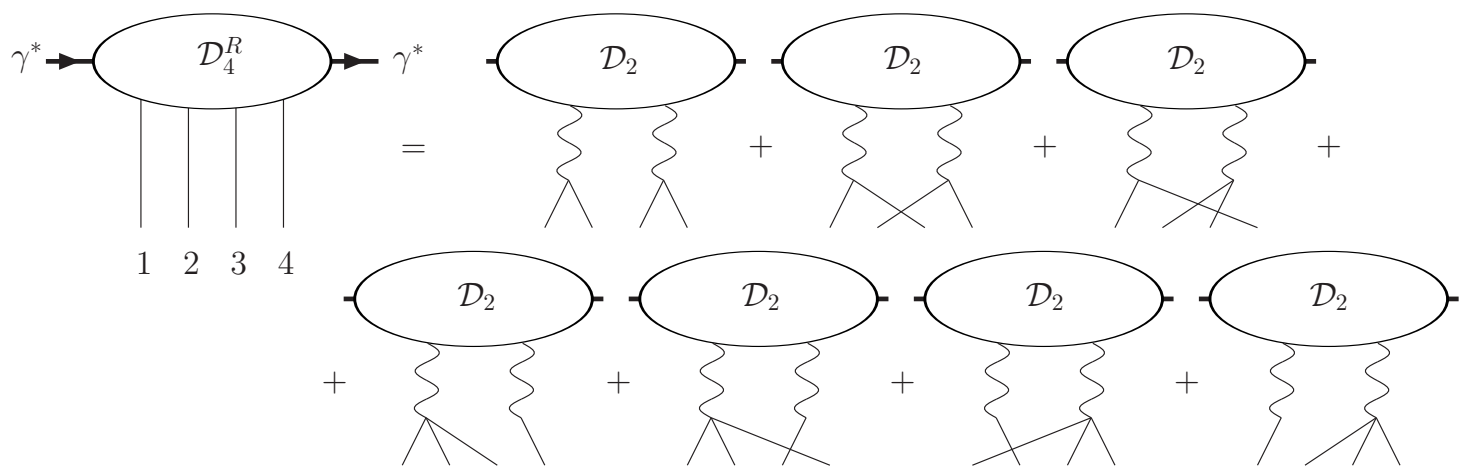

Figure 6: Decomposition of the reggeizing part of the four reggeon amplitude $\mathcal{D}_{4}^{R}$ in term of reggeized gluons.

listed in the AGK paper.

Starting from these functions $\mathcal{D}_{4}^{I}$ and $\mathcal{D}_{4}^{R}$, the investigation in [6] has shown in some detail how the AGK counting rules work in pQCD: the analysis has to be done seperately for $\mathcal{D}_{4}^{I}$ and $\mathcal{D}_{4}^{R}$. For the first piece, we obtain the counting arguments for the Pomerons (which is even-signatured) given by AGK; here the essential ingredient is the complete symmetry of $\mathcal{D}_{4}^{I}$ under the permutation of reggeized gluons. In the latter piece, the odd-signature reggeizing gluons lead to counting rules which are slightly different from those of the even signature Pomeron: once the bootstrap properties have been invoked and $\mathcal{D}_{4}^{R}$ is expressed in terms of $\mathcal{D}_{2}$ functions, cutting lines through the reggeized gluon appear. Since it carries negative signature, the relative weight between cut and uncut reggeon is different from the Pomeron. It should be stressed, however, that the generalization of the AGK analysis to odd signature reggeons is contained in the AGK paper; in pre-QCD times, however, there was no obvious reason for considering Regge poles other than the Pomeron.

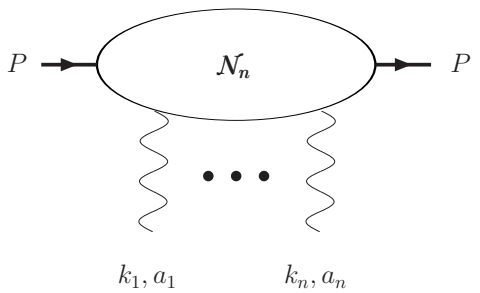

Figure 7: Graphical illustration of the coupling $\mathcal{N}_{n}\left(\boldsymbol{k}_{1}, a_{1} ; \ldots ; \boldsymbol{k}_{n}, a_{n} ; \omega\right)$ of a proton to $n$ reggeized gluons.

Let us now turn to the main goal of this paper, to the use of the pQCD cutting rules in a nonperturbative environment (e.g.multi-ladder exchanges in $p p$ scattering). Basic ingredients are the nonperturbative couplings of $n$ reggeized gluons to the proton. In order to justify the use of pQCD we need a hard scattering subprocess: we will assume that all reggeized gluons are connected to some hard scattering subprocess; consequently, each gluon line will have its transverse momentum in the kinematic region where the use of pQCD can be justified. Since AGK applies to the high energy limit (i.e. the small- $x$ region), all $t$-channel gluons are reggeized. Based upon the analysis in pQCD, we formulate a few general conditions which the nonperturbative couplings have to satisfy. Following the original AGK paper, we will denote these couplings by $\mathcal{N}_{n}\left(\boldsymbol{k}_{1}, a_{1} ; \ldots ; \boldsymbol{k}_{n}, a_{n} ; \omega\right)$, where $\boldsymbol{k}_{i}$ and $a_{i}$ refer to transverse 
momenta and color of the $i$ th gluon, and require that

(i) they are symmetric under the simultaneous exchange of momenta and color, e.g. $\left(k_{i}, a_{i}\right) \leftrightarrow\left(k_{j}, a_{j}\right)$;

(ii) cut and uncut vertices are identical, independently where the cut line enters.

In the following sections we will work out a few results which follow from these conditions.

We conclude this section with a remark on the reggeizing pieces, $D_{4}^{R}$. The analysis of $\mathcal{D}_{2}$, $\mathcal{D}_{3}$, and $\mathcal{D}_{4}$ in [7], and of $\mathcal{D}_{5}$ and $\mathcal{D}_{6}$ in [8] lead to the following conjecture about the general coupling of $n$ reggeized gluons to virtual photons. Functions with an even number of lines, $\mathcal{D}_{2 n}$, contain

- a piece which is completely symmetric, $\mathcal{D}_{2 n}^{I}$;

- a second piece which can be expressed in terms of $\mathcal{D}_{2 n-2}$, by either having two of the outgoing reggeized gluons each split into two gluons or one of the outgoing gluons split into three gluons;

- a third piece which is reduced to $\mathcal{D}_{2 n-4}$ with further splittings at the lower end etc.

Finally, functions with an odd number, $\mathcal{D}_{2 n+1}$,

- can always be reduced to $\mathcal{D}_{2 n}$, with one splitting at the lower end.

As a consequence, also these reggeizing pieces can be reduced to the couplings $\mathcal{N}_{2 n}$. Since the reggeization of the gluon is a general feature of QCD, valid to all orders in perturbation theory, the appearance of these reggeizing pieces can be expected to happen also in a nonperturbative coupling; in other words, in addition to the functions $\mathcal{N}_{2 n}$ mentioned before, we expect also contributions with the same functions $\mathcal{N}_{2 n}$, but one (or more) of the reggeized gluons split into two gluons etc.

In the following we will apply the AGK analysis to $p p$ scattering, assuming that the couplings of $n$ gluons to the proton satisfy the requirements listed above. In this paper we will not analyse yet the reggeizing pieces; their analysis will be taken up in a future paper. Another application of the AGK analysis is DIS, in particular the production of diffractive final states. Such an analysis cannot avoid to include the reggeizing pieces: to lowest order in $\alpha_{s}$ the coupling of $n$ gluons to the virtual photon consists of reggeizing pieces only and hence cannot be ignored.

\section{Inclusive cross section}

In this section we discuss the AGK rules for inclusive cross sections. Since we are not asking for a final state which provides a hard scale, such a discussion might seem somewhat academic: rescattering in $p p$ collisions is described by multiple exchanges of nonperturbative Pomerons, not simply of bound states of reggeized gluons. For consistency, however, it is important to show that BFKL exchanges lead to the same counting rules as the nonpertubative Pomerons. Also, for the modelling of events with multiple scattering events, it will be useful to illustrate the pattern of AGK cancellations.

\subsection{The nonperturbative case}

Let us begin by recapitulating the counting rules of the original AGK paper. The contribution of the $n$ Pomeron cut to the scattering amplitude is given by

$$
\mathcal{T}_{A B}^{\text {n-cut }}(s, t)=\int \frac{d \omega}{2 i} \xi(\omega) s^{1+\omega} \operatorname{disc}_{\omega}^{(n)}[\mathcal{F}(\omega, t)]
$$


and the discontinuity of the partial wave has the form given in (3). Doing the $\omega$ integral we arrive at:

$$
\mathcal{T}_{A B}^{\text {n-cut }}(s, t)=\pi \int \frac{d \Omega_{n}}{n !} \gamma_{\left\{\beta_{j}\right\}} \xi(\beta) s^{1+\beta} \mathcal{N}_{n}^{A} \mathcal{N}_{n}^{B}
$$

where we have defined $\beta \equiv \beta\left(\left\{\boldsymbol{k}_{j}\right\}\right)=\sum_{j=1}^{n} \beta_{j}$ and $\mathcal{N}_{n}^{A, B} \equiv \mathcal{N}_{n}^{A, B}\left(\left\{\boldsymbol{k}_{j}\right\} ; \beta\right)$. The energy discontinuity (imaginary part) is obtained by simply replacing the signature factor (2) by 1. As we have explained before, the aim of AGK is to obtain the same result for the discontinuity from the unitarity equation, i.e. from products of production amplitudes. Each such contribution can graphically be illustrated by a cutting line (Fig.3). One of the main results of AGK states that the $s$-discontinuity of the $n$-cut contribution to the amplitude, $\operatorname{disc}_{s}\left[\mathcal{T}_{A B}^{\text {n-cut }}(s, t)\right]$, can be written as a sum over the number $k$ of cut pomerons $(k=1, \ldots, n)$,

$$
\mathcal{A}^{n}(s, t) \stackrel{\text { def }}{=} \operatorname{disc}_{s}\left[\mathcal{T}_{A B}^{\mathrm{n} \text {-cut }}(s, t)\right]=\sum_{k=0}^{n} \mathcal{A}_{k}^{n}(s, t),
$$

and the terms in the sum are

$$
\mathcal{A}_{k}^{n}(s, t)=2 \pi i \int \frac{d \Omega_{n}}{n !} \mathcal{F}_{k}^{n} s^{1+\beta} \mathcal{N}_{n}^{A} \mathcal{N}_{n}^{B},
$$

where we have introduced the AGK factors

$$
\mathcal{F}_{k}^{n}=\left\{\begin{array}{ll}
(-1)^{n} 2^{n-1}+\gamma_{\left\{\beta_{j}\right\}} & \text { if } k=0 \\
(-1)^{n-k} 2^{n-1}\left(\begin{array}{c}
n \\
k
\end{array}\right) & \text { if } k>0
\end{array} .\right.
$$

If a (nonperturbative) pomeron is viewed as a multiperipheral chain of secondary particles, the cut of each pomeron gives a uniform distribution in rapidity, and the sum in (8) leads to density fluctuations.

The simplest case, the two-Pomeron exchange, has the three contributions illustrated in Fig.8: Figure 8.a shows the diffractive cut: all the pomerons are left uncut, and there is a

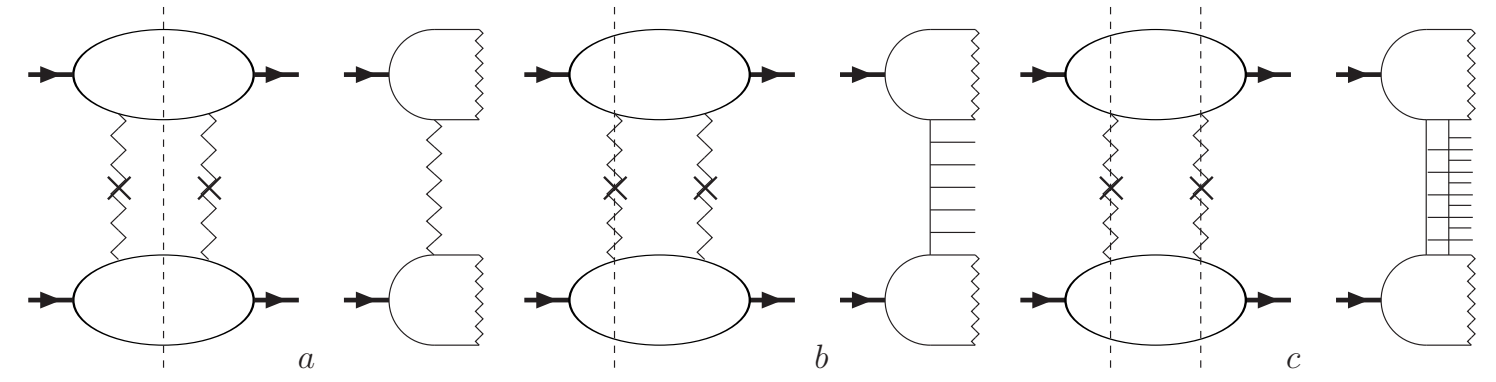

Figure 8: There are three different ways to cut the two-pomerons diagram: a) diffractive cut, b) single multiplicity cut and c) double multiplicity cut.

rapidity gap between the fragmentation regions of the two particles. Figure 8.b represents a single multiplicity cut in which only one pomeron has been cut. Eventually, the situation shown in figure 8.c corresponds to two cut pomerons, and the multiplicity of particles is doubled with respect to the previous case. Neglecting the real part of the pomeron signature factor (2) it reduces to the imaginary unit $i$, and the $\gamma$ factor (4) is just $(-1)^{n-1}$. From (10) we obtain for the weight factors the well-known results:

$$
\begin{array}{rcc}
1: & \text { diffractive } & (k=0) \\
-4: & \text { single multiplicity } & (k=1) \\
2: & \text { double multiplicity } & (k=2) .
\end{array}
$$


In other words, the different contributions are in the proportion

$$
\mathcal{A}_{0}^{2}: \mathcal{A}_{1}^{2}: \mathcal{A}_{2}^{2}=1:-4: 2 .
$$

This result can be summarized as follows: final states with a rapidity gap $(k=0)$ are accompanied by other final states with double density $(k=2)$, and their respective cross sections come with the relative weight given by (12). At the same time, these final states are connected with corrections to the cross section of final states with normal density $(k=1)$, as contained in (12).

For later use we note an important generalization: suppose we substitute one of the soft pomerons by a different Regge pole, and we concentrate on those contributions where this Regge pole is cut (later on, in the context of inclusive jet production, we shall apply this argument to a hard cut gluon ladder). Following the previous argument used to derive the usual AGK rules, we simply sum over cut and uncut soft pomerons, where the uncut soft Pomerons appear on both the left and the right side of the cut reggeon. As a result, the $\gamma$ factor in the first line of (10) does not appear, and for the contribution of $k$ cut Pomerons we simply obtain:

$$
\mathcal{F}_{k}^{n} \propto(-1)^{n-1-k}\left(\begin{array}{c}
n-1 \\
k
\end{array}\right), \quad k=0, \ldots, n-1
$$

Clearly the sum over $k$ vanishes identically: soft multi-Pomeron corrections to a single cut Regge pole cancel. This argument is easily generalized to two or more singled out cut Regge poles.

So far we have discussed the AGK counting only at one rapidity value. The AGK paper also addresses the question of how to continue the $s$-discontinuity cutting lines inside the upper or lower vertex function $\mathcal{N}_{n}$. When following, for example, the cut Pomerons inside $\mathcal{N}_{n}$ (or cutting lines running between Pomerons) one faces the question of how these cutting lines pass through Pomeron interaction vertices ('cut vertices'). AGK constraints can be formulated only for a very restricted class of interaction vertices (in particular, for the $1 \rightarrow n$ Pomeron vertex). For the general case (for example, for the $2 \rightarrow 2$ vertex) this is not the case; only explicit models, e.g. calculations in pQCD, can provide further information.

\subsection{The $\mathrm{pQCD}$ case}

Let us now derive the counting rule (10) in pQCD. Since a Pomeron appears as a bound state of two reggeized gluons, the discussion of $n$ Pomerons has to start from $2 n$ reggeized gluons. Moreover, in LLA the signature factor of the reggeized gluon is real, $\xi_{\mathbb{G}}(\boldsymbol{k})=-2 / \pi \boldsymbol{k}^{2}$; therefore, working in LLA, the diagrams with any cut reggeized gluon are suppressed and will not be considered. The derivation of (10) follows from straightforward combinatorics. The simplest case, $n=2$, has been discussed in [6], and we can use these results (Fig.9) for illustrating the general proof.

The situation is depicted in figure 9: each term denotes the product of production amplitudes, and it is understood that we sum and integrate over the produced gluons. We again take the discontinuity in $\omega$ across the four $t$-channel gluons (denoted by crosses), and all the produced gluons are absorbed inside the blobs above and below. In the first diagram, the production amplitude on the left of the cutting line (vertical dashed line) contains the exchange of one gluon, the amplitude on the rhs three gluons. Three gluon exchange comes with a negative sign (from the $\gamma$-factor in (3) and from the signature factor in (1)); moreover, there is a symmetry factor 1/3!. Similarly, the second term in Fig.9 denotes the square of two production amplitudes: two-gluon exchange is purely imaginary and has the symmetry factor $1 / 2$ !. Note that, in contrast to the 2 pomeron exchange discussed above, in the 


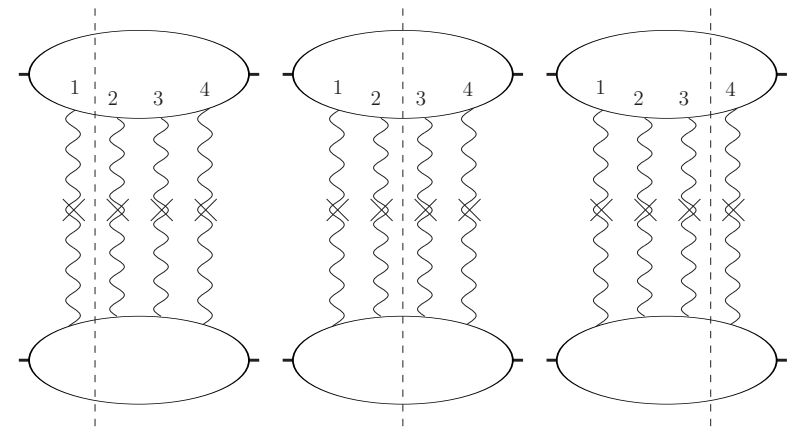

Figure 9: The three $s$-channel cuts contributing to the four reggeized gluon exchange.

case of reggeized gluons we do not need to consider cutting lines inside the reggeized gluons: compared to an uncut gluon, a cut gluon line is suppressed in order $\alpha_{s}$.

Since we are looking for the contributions of BFKL Pomerons which are bound states of two gluons, we must consider all possible pairings among the reggeized gluons; using the notation $\left(i_{1} j_{1}\right)\left(i_{2} j_{2}\right)$ to indicate that gluon $i_{1}$ forms a bound state with the gluon $j_{1}$, and gluon $i_{2}$ with $j_{2}$, the three possibilities are: (12)(34), (13)(24) and (14)(23).

Let us first consider the 'diagonal' configuration, in which the pairings in the upper and in the lower blob are identical. One easily sees that these different configurations contribute with different multiplicities. Starting with the first graph in Fig.9, in all three possible pairings one of the gluon pairs is cut, and the other one is not. This means that all the three configurations contribute with single multiplicity $(k=1)$; the weight factor is $-3 \times 1 / 3$ !. The same argument holds for the third graph in Fig.9. In the second graph, the configuration (12)(34) does not have any cut pair; this contributes to the diffractive term $(k=0)$, and the weight is $(1 / 2 !) \times(1 / 2 !)=1 / 4$. The other two pairings have both gluon pairs cut, and therefore they contribute to the double multiplicity term $(k=2)$; the weight factor is $2 \times(1 / 2 !) \times(1 / 2 !)=1 / 2$. This agrees with the AGK result $(12)$.

It should be clear how to generalize this counting to the exchange of $2 n$ reggeized gluons. The general result for the contribution of $k$ cut Pomerons assumes the form (for the explicit computation see Appendix A):

$$
\tilde{\mathcal{A}}_{k}^{n}(s, t)=2 \pi i \tilde{\mathcal{F}}_{k}^{n} \int \frac{d \Omega_{2 n}}{n !} s^{1+\tilde{\beta_{n}}} \mathcal{N}_{2 n} \mathcal{N}_{2 n} \tilde{\gamma}_{\left\{\boldsymbol{k}_{j}\right\}},
$$

where

$$
\tilde{\mathcal{F}}_{k}^{n}=\left\{\begin{array}{ll}
(-1)^{n} 2^{n-1}+(-1)^{n-1} & \text { if } k=0 \\
(-1)^{n-k} 2^{n-1}\left(\begin{array}{l}
n \\
k
\end{array}\right) & \text { if } k>0
\end{array} .\right.
$$

and

$$
\tilde{\gamma}_{\left\{\boldsymbol{k}_{j}\right\}} \stackrel{\text { def }}{=} \frac{2^{1-n}}{\pi} \prod_{j=1}^{2 n} \xi_{\mathbb{G}}\left(\boldsymbol{k}_{j}\right)=\frac{2^{1-n}}{\pi} \prod_{j=1}^{2 n} \frac{2}{\pi \boldsymbol{k}_{j}^{2}} .
$$

Here we use the tilde symbol to indicate that the result is obtained in pQCD. Note that the weight factors $\tilde{\mathcal{F}}_{k}^{n}$ coincide with those defined in (10) for $k \neq 0$, while for $k=0$ they coincide when the real part of the soft pomeron signature factor is neglected $\left(\gamma_{\left\{\beta_{j}\right\}}=(-1)^{n-1}\right)$.

It should be stressed that this discussion has made use only of the very general symmetry properties of the 4-gluon vertex functions above and below the four gluon state. Each vertex function can very well have a complicated internal structure of reggeized gluons; for example, it may consist of a single BFKL ladder which splits into four gluons. In this case, our analysis 
has demonstrated the AGK rules inside a closed Pomeron loop. The crucial ingredient is the symmetry structure, and in this example it is garanteed by the form of the $2 \rightarrow 4$ gluon vertex. In an anologous way one can generalize the validity of the AGK analysis to general reggeon diagrams in $\mathrm{pQCD}$.

What remains is the discussion [6] of the case where, in Fig.9, the pairings above and below the four gluon state do not match (non-diagonal configurations): an example is given in Fig.10a. The corresponding final states are depictured in Fig.10b. Here the situation is the following: in the upper rapidity interval we have multiplicity $k$ which can take one of the values 0,1 , or 2 ( $k=0$ in our example). Each multiplicity $k$ comes with a relative weight, described by the three-component vector $(1,-4,2)$. In the lower rapidity interval we have multiplicity $k^{\prime}$ (in our example $k^{\prime}=2$ ). Contributions to this $k^{\prime}$ can come from different $k$ in the upper interval, so the transition from the upper to the lower interval - named a 'switch' - can be described by a $3 \times 3$ matrix, whose elements are defined to the fraction of configurations that lead from $k$ to $k^{\prime}$ :

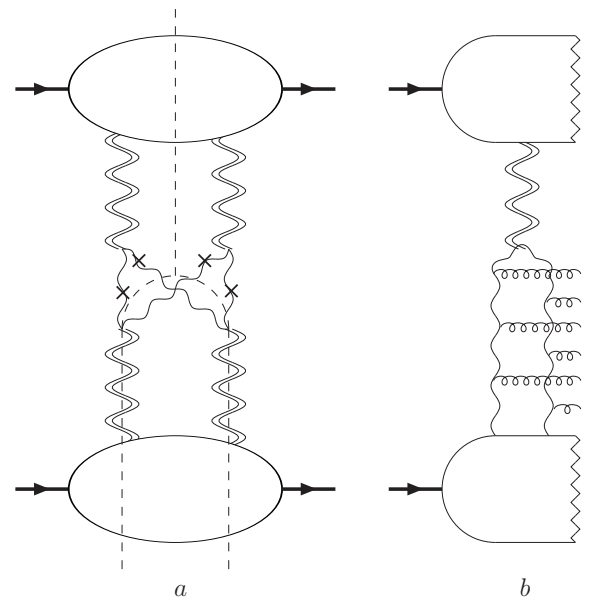

Figure 10: An example of non-diagonal configuration: a double wavy line represent a two reggeized gluons bound state (they can be freely moved due to the symmetry of the impact factors). The bound state configuration is not the same above and below the cut. In a) the cut forward amplitude is shown, while in b) we depict an example of a final state coming from the cut.

$$
\mathcal{M}=\left(\begin{array}{ccc}
0 & 0 & \frac{1}{2} \\
0 & 1 & 0 \\
1 & 0 & \frac{1}{2}
\end{array}\right)
$$

(here $k$ labels the columns, $k^{\prime}$ the rows). Now one easily verifies that the vector $\mathcal{F}^{t}=(1,-4,2)$ is eigenvector of this matrix $\mathcal{M}$ : this implies that the ratios: diffraction : single density : double density holds for both the upper and the lower rapidity interval, i.e. it is invariant.

This pattern holds for an arbitrary number, $2 n$, of exchanged gluons. The number $k$ of cut BFKL Pomerons denotes the density of gluons in the final state, and for diagonal configurations the AGK factors given in (15) measure the relative weight of states with density $k$. If we move to the neighbouring rapidity interval, the density changes to $k^{\prime}$ : the transitions from $k$ to $k^{\prime}$ define a matrix $\mathcal{M}$, and the eigenvectors of this matrix are formed by the AGK factors in (15). They define, for each rapidity interval, the relative weight of density $k$. Some details are given in the Appendix. 


\subsection{An example}

More detailed results can be derived if one assumes a specific model for the couplings $\mathcal{N}_{2 n}\left(k_{1}, a_{1} ; \ldots ; k_{2 n}, a_{2 n} ; \omega\right)$ to hadrons $A$ and $B$. A popular choice is the eikonal model. In order to satisfy our symmetry requirements, we have to start from the ansatz:

$$
\begin{aligned}
& \mathcal{N}_{2 n}^{A}\left(\boldsymbol{k}_{1}, a_{1} ; \ldots ; \boldsymbol{k}_{2 n}, a_{2 n} ; \omega\right)= \\
& \frac{1}{\sqrt{\left(N_{c}^{2}-1\right)^{n}}}\left(\phi^{A}\left(\boldsymbol{k}_{1}, \boldsymbol{k}_{2} ; \omega_{12}\right) \delta_{a_{1} a_{2}} \cdot \ldots \cdot \phi^{A}\left(\boldsymbol{k}_{2 n-1}, \boldsymbol{k}_{2 n} ; \omega_{2 n-1,2 n}\right) \delta_{a_{2 n-1} a_{2 n}}+\sum_{\text {Permutations }}\right) .
\end{aligned}
$$

Here $\omega_{12}=\omega_{1}+\omega_{2}$, and each factor $\phi^{A}\left(k_{1}, k_{2} ; \omega_{12}\right) \delta_{a_{1} a_{2}}$ represents a BFKL amplitude in the color singlet state, convoluted with an impact factor $\phi_{0}^{A}\left(\boldsymbol{k}_{1}, \boldsymbol{k}_{2}\right)$. The sum has to extend over all possible pairings of the gluons $1, \ldots, 2 n:\left(i_{1}, j_{1}\right), \ldots,\left(i_{n}, j_{n}\right)$ (alltogether, there are $C^{n}=(2 n-1)$ !! such possibilities). When inserting this ansatz for $\mathcal{N}_{2 n}^{A}$ and $\mathcal{N}_{2 n}^{B}$ into (42), (43) and performing the counting described in the first subsection of the Appendix we proceed in the following way:

(a) we rewrite (42) in the same way as (3), i.e. we define the Sommerfeld-Watson transformation $\tilde{\mathcal{F}}^{n}(\omega, \boldsymbol{q})$ of the scattering amplitude $\tilde{\mathcal{T}}^{n}(s, \boldsymbol{q})$ and take the discontinuity across the $2 n$-reggeon cut:

$$
\operatorname{disc}_{\omega} \tilde{\mathcal{F}}^{n}(\omega, \boldsymbol{q})=4 i \sum_{j=1}^{2 n-1} S_{j}^{n} \int d \Omega_{2 n} \mathcal{N}_{2 n}^{A} \delta\left(\omega-\Sigma_{j} \beta_{j}\right) \mathcal{N}_{2 n}^{B} \prod_{j} \xi_{\mathbb{G}}\left(\boldsymbol{k}_{j}\right) .
$$

(b) when combining the $\mathcal{N}_{2 n}$ from above and from below, we retain only the diagonal combinations, i.e. those combinations where the pairings above and below match. In this way we sum over all bound states. Formally, this coincides with the large- $N_{c}$ limit. Each term obtained in this way is a product of $n$ BFKL exchanges.

(c) in each of these products of $n$ BFKL exchanges we have to apply the discussion given after eq.(4): for each pair of gluons $(i j)$ we define the variable $\omega_{i j}$ and we substitute $\delta\left(\omega-\sum \beta_{j}\right) \rightarrow$ $\left[\prod_{(i j)} \int d \omega_{i j} \delta\left(\omega_{i j}-\beta_{i}-\beta_{j}\right)\right] \delta\left(\omega-\sum \omega_{i j}\right)$. In order to take care of momentum conservation we write the measure as

$$
d \Omega_{2 n}=\int d^{2} \boldsymbol{b} e^{i \boldsymbol{b} \cdot \boldsymbol{q}} \prod_{j=1}^{2 n} \frac{d^{2} \boldsymbol{k}_{j}}{(2 \pi)^{2}} e^{-i \boldsymbol{b} \cdot \boldsymbol{k}_{j}} .
$$

The discontinuity then assumes the form:

$$
\begin{array}{r}
\operatorname{disc}_{\omega} \tilde{\mathcal{F}}^{n}(\omega, t)=4 i \sum_{j=1}^{2 n-1} S_{j}^{n} C^{n} \int d^{2} \boldsymbol{b} e^{i \boldsymbol{b} \cdot \boldsymbol{q}} \prod_{(i j)}\left[\int \frac{d^{2} \boldsymbol{k}_{i}}{(2 \pi)^{2}} \int \frac{d^{2} \boldsymbol{k}_{j}}{(2 \pi)^{2}} e^{-i \boldsymbol{b} \cdot\left(\boldsymbol{k}_{i}+\boldsymbol{k}_{j}\right)}\right. \\
\left.\cdot \int d \omega_{i j} \xi_{\mathbb{G}}\left(\boldsymbol{k}_{i}\right) \xi_{\mathbb{G}}\left(\boldsymbol{k}_{j}\right) \phi^{A}\left(\boldsymbol{k}_{i}, \boldsymbol{k}_{j}, \omega_{i j}\right) \delta\left(\omega_{i j}-\beta_{i}-\beta_{j}\right) \phi^{B}\left(\boldsymbol{k}_{i}, \boldsymbol{k}_{j}, \omega_{i j}\right)\right] \delta\left(\omega-\Sigma_{(i j)} \omega_{i j}\right) .
\end{array}
$$

(d) Defining the variable

$$
\boldsymbol{q}_{i j}=\boldsymbol{k}_{i}+\boldsymbol{k}_{j},
$$

using the result (49) of the Appendix and observing that, since $\xi_{\mathbb{G}}(\boldsymbol{k})$ is real, $\xi_{\mathbb{G}}\left(\boldsymbol{k}_{i}\right) \xi_{\mathbb{G}}\left(\boldsymbol{k}_{j}\right)=$ $\Im\left[-i\left(i \xi_{\mathbb{G}}\left(\boldsymbol{k}_{i}\right)\right)\left(i \xi_{\mathbb{G}}\left(\boldsymbol{k}_{j}\right)\right)\right]=\gamma_{2}\left(\boldsymbol{k}_{i}, \boldsymbol{k}_{j}\right)$, we can write the multiplicity $\mathrm{k}$ (for $k>0$ ) contribution to $(21)$ in the following form:

$$
\operatorname{disc}_{\omega} \tilde{\mathcal{F}}_{k}^{n}(\omega, t)=4 i \frac{(-1)^{n-k}}{k !(n-k) !} \int d^{2} \boldsymbol{b} e^{i \boldsymbol{b} \cdot \boldsymbol{q}} \prod_{(i j)}\left[\int \frac{d^{2} \boldsymbol{q}_{i j}}{(2 \pi)^{2}} e^{-i \boldsymbol{b} \cdot \boldsymbol{q}_{i j}} \int d \omega_{i j}\right.
$$




$$
\left.\int \frac{d^{2} \boldsymbol{k}_{i}}{(2 \pi)^{2}} \gamma_{2}\left(\boldsymbol{k}_{i}, \boldsymbol{k}_{j}\right) \phi^{A}\left(\boldsymbol{k}_{i}, \boldsymbol{q}_{i j}-\boldsymbol{k}_{i}, \omega_{i j}\right) \delta\left(\omega_{i j}-\beta_{i}-\beta_{j}\right) \phi^{B}\left(\boldsymbol{k}_{i}, \boldsymbol{q}_{i j}-\boldsymbol{k}_{i}, \omega_{i j}\right)\right] \delta\left(\omega-\Sigma_{(i j)} \omega_{i j}\right) \text {. }
$$

(e) Comparing the integrand of the $\omega_{i j}$ integral with formula (3), we identify it as the discontinuity in $\omega_{i j}$ of the partial wave of the BFKL pomeron across the two-reggeon cut:

$$
\begin{array}{r}
\int \frac{d^{2} \boldsymbol{k}_{i}}{(2 \pi)^{2}} \gamma_{2}\left(\boldsymbol{k}_{i}, \boldsymbol{k}_{j}\right) \phi^{A}\left(\boldsymbol{k}_{i}, \boldsymbol{q}_{i j}-\boldsymbol{k}_{i}, \omega_{i j}\right) \delta\left(\omega_{i j}-\beta_{i}-\beta_{j}\right) \phi^{B}\left(\boldsymbol{k}_{i}, \boldsymbol{q}_{i j}-\boldsymbol{k}_{i} ; \omega_{i j}\right)= \\
\frac{1}{\pi i} \operatorname{disc}_{\omega_{i j}}\left[\mathcal{F}_{B F K L}\left(\omega_{i j}, \boldsymbol{q}_{i j}\right)\right]
\end{array}
$$

where

$$
\mathcal{F}_{B F K L}\left(\omega_{i j}, \boldsymbol{q}_{i j}\right)=\int \frac{d^{2} \boldsymbol{k} d^{2} \boldsymbol{k}^{\prime}}{(2 \pi)^{4}} \phi_{0}^{A}\left(\boldsymbol{k}, \boldsymbol{q}_{i j}-\boldsymbol{k}\right) \mathcal{G}_{2}\left(\boldsymbol{k}, \boldsymbol{q}_{i j}-\boldsymbol{k}, \boldsymbol{k}^{\prime}, \boldsymbol{q}_{i j}-\boldsymbol{k}^{\prime} ; \omega_{i j}\right) \phi_{0}^{B}\left(\boldsymbol{k}^{\prime}, \boldsymbol{q}_{i j}-\boldsymbol{k}^{\prime}\right) .
$$

Therefore (23) becomes

$$
\begin{array}{r}
\operatorname{disc}_{\omega} \tilde{\mathcal{F}}_{k}^{n}(\omega, t)=4 i \frac{(-1)^{n-k}}{k !(n-k) !} \int d^{2} \boldsymbol{b} e^{i \boldsymbol{b} \cdot \boldsymbol{q}} \\
\cdot \prod_{(i j)}\left[\frac{1}{\pi i} \int \frac{d^{2} \boldsymbol{q}_{i j}}{(2 \pi)^{2}} e^{-i \boldsymbol{b} \cdot \boldsymbol{q}_{i j}} \int d \omega_{i j} \operatorname{disc}_{\omega_{i j}}\left[\mathcal{F}_{B F K L}\left(\omega_{i j}, \boldsymbol{q}_{i j}\right)\right]\right] \delta\left(\omega-\Sigma_{(i j)} \omega_{i j}\right) .
\end{array}
$$

(f) Returning to the energy representation:

$\tilde{\mathcal{A}}_{k}^{n}(s, t)=4 i s \frac{(-1)^{n-k}}{k !(n-k) !} \int d^{2} \boldsymbol{b} e^{i \boldsymbol{b} \cdot \boldsymbol{q}}\left[\frac{1}{\pi i} \int \frac{d^{2} \boldsymbol{q}^{\prime}}{(2 \pi)^{2}} e^{-i \boldsymbol{b} \cdot \boldsymbol{q}^{\prime}} \int d \omega^{\prime} \operatorname{disc}_{\omega^{\prime}}\left[\mathcal{F}_{B F K L}\left(\omega^{\prime}, \boldsymbol{q}^{\prime}\right)\right] s^{\omega^{\prime}}\right]^{n}$

and defining:

$$
\Omega(s, \boldsymbol{b})=\frac{1}{\pi i} \int \frac{d^{2} \boldsymbol{q}}{(2 \pi)^{2}} e^{-i \boldsymbol{b} \cdot \boldsymbol{q}} \int d \omega \operatorname{disc}_{\omega}\left[\tilde{\mathcal{F}}_{B F K L}^{n}(\omega, \boldsymbol{q})\right] s^{\omega}
$$

we obtain, after summation over $n \geq k$,

$$
\tilde{\mathcal{A}}_{k}(s, t)=4 i s \int d^{2} \boldsymbol{b} e^{i \boldsymbol{b} \cdot \boldsymbol{q}} P(s, \boldsymbol{b}),
$$

where

$$
P(s, \boldsymbol{b})=\frac{[\Omega(s, \boldsymbol{b})]^{k}}{k !} e^{-\Omega(s, \boldsymbol{b})}
$$

is the probability of having $k$ cut Pomerons at fixed impact parameter $b$.

\section{Single and double inclusive cross sections}

In this section we turn to the realistic case, where one ore more hard final states (e.g. jets or heavy flavors) are produced. Again we begin with a brief repeat of the AGK results and then turn to pQCD.

In the AGK paper it has been shown that, for the single (or double) particle inclusive cross section, large classes of multi-pomeron corrections cancel. We illustrate this result in Fig.11: for the single inclusive case (Fig.11a) all multi-Pomeron exchanges across the produced particle cancel (Fig.11b), and the same is true for the double inclusive case (Fig.12a, 

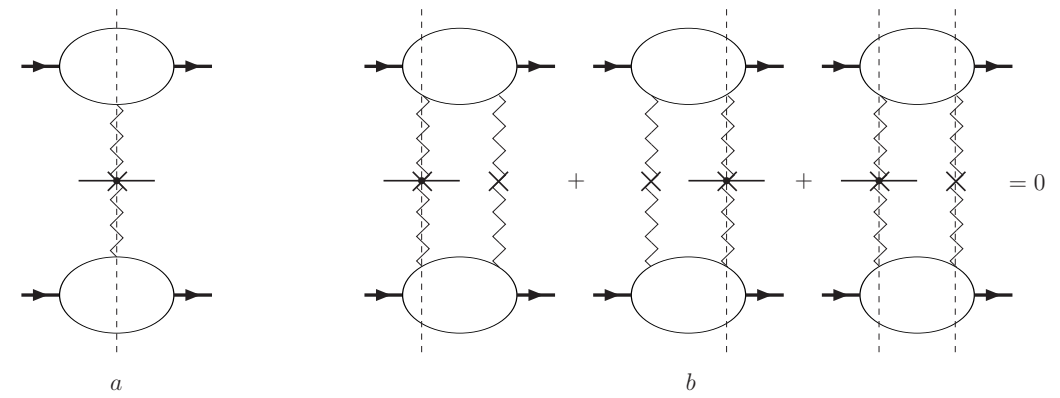

Figure 11: The pattern of cancellation is shown for the single jet inclusive case: the first graph survives, the three other cancel each other.
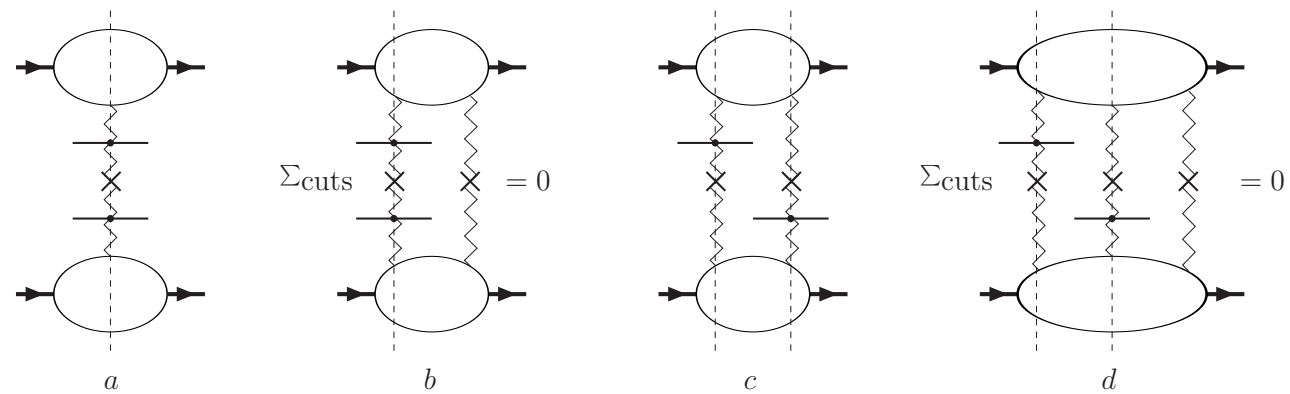

Figure 12: The pattern of cancellation is shown for the double jet inclusive case: the graph a and c contribute, the other interfere destructively giving a vanishing contribution.

12b). For the latter case, however, there is a new contribution: the produced particles originate from different Pomerons (Fig.12c). This term is of particular interest, since it introduces longe range correlations in the rapidity difference $y_{1}-y_{2}$ :

$$
\rho\left(y_{1}, y_{2}\right) \sim \frac{1}{\sigma_{t o t}} \frac{d^{2} \sigma}{d y_{1} d y_{2}}-\frac{1}{\sigma_{t o t}^{2}} \frac{d \sigma}{d y_{1}} \frac{d \sigma}{d y_{2}}
$$

where, for simplicity, we have suppressed all variables other than the rapidities. The multiPomeron corrections to this term, again, cancel (Fig.12d). In all cases, however, there remain multipomeron corrections between the production vertices and the projectiles. Examples are illustrated in Fig.13.
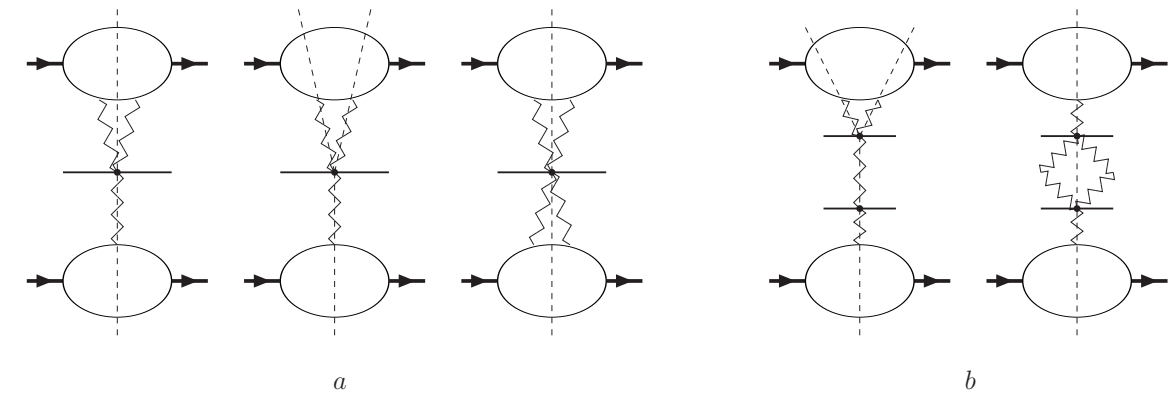

Figure 13: Some examples of the terms that survive to the AGK cancellation for the single jet production a) and for the double jet production $\mathrm{b}$ ). 


\subsection{Inclusive single jet production in pQCD}

We now turn to the corresponding final states in pQCD. As usual, the presence of the hard scale in the final state justifies, as far as the cut ladder with the produced jet or heavy flavor state is concerned, the use of perturbation theory. The basic process is illustrated in Fig.14:

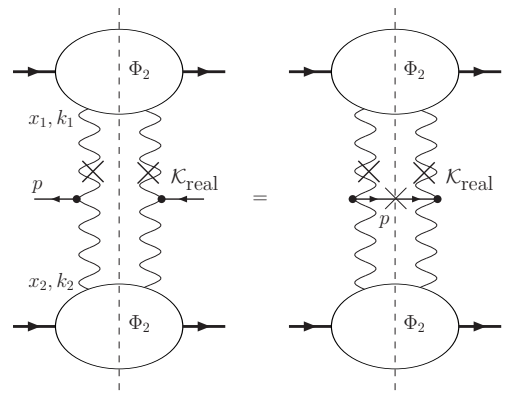

Figure 14: Pictorial representation of the basic process for the single inclusive jet production.

in the context of LL BFKL ladders (or $k_{t}$ factorization), the cross section has the form:

$$
\begin{gathered}
\frac{d \sigma}{d y d^{2} \boldsymbol{p}}(y, \boldsymbol{p})=\frac{1}{4} \int \frac{d^{2} \boldsymbol{k}_{1}}{(2 \pi)^{2}} \int \frac{d^{2} \boldsymbol{k}_{2}}{(2 \pi)^{2}}(2 \pi)^{2} \delta^{(2)}\left(\boldsymbol{p}-\boldsymbol{k}_{1}-\boldsymbol{k}_{2}\right) \\
\frac{\Phi_{2}\left(x_{1} ; \boldsymbol{k}_{1},-\boldsymbol{k}_{1}\right)}{\boldsymbol{k}_{1}^{2} \boldsymbol{k}_{1}^{2}} \mathcal{K}_{\text {real }}\left(\boldsymbol{k}_{1},-\boldsymbol{k}_{1} ;-\boldsymbol{k}_{2}, \boldsymbol{k}_{2}\right) \frac{\Phi_{2}\left(x_{2} ; \boldsymbol{k}_{2},-\boldsymbol{k}_{2}\right)}{\boldsymbol{k}_{2}^{2} \boldsymbol{k}_{2}^{2}}
\end{gathered}
$$

with $x_{1}$ and $x_{2}$ being the momentum fractions of the incoming gluons with momenta $\boldsymbol{k}_{1}$ and $\boldsymbol{k}_{2}$, the rapidity of the emitted jet given by $y=1 / 2 \log \left(x_{1} / x_{2}\right)$, and $\mathcal{K}_{\text {real }}$ is the real emission BFKL kernel (Lipatov vertex) in the forward direction,

$$
\mathcal{K}_{\text {real }}\left(\boldsymbol{k}_{1},-\boldsymbol{k}_{1} ;-\boldsymbol{k}_{2}, \boldsymbol{k}_{2}\right)=\frac{4 \alpha_{s} N_{c}}{(2 \pi)^{2}} \frac{\boldsymbol{k}_{1}^{2} \boldsymbol{k}_{2}^{2}}{\left(\boldsymbol{k}_{1}+\boldsymbol{k}_{2}\right)^{2}} .
$$

The connection between the unintegrated gluon densities $\Phi_{2}(x, \boldsymbol{k},-\boldsymbol{k})$ and the usual gluon density $g\left(x, Q^{2}\right)$ is given by:

$$
x g\left(x, Q^{2}\right)=\int_{Q_{0}^{2}}^{Q^{2}} \frac{d^{2} \boldsymbol{k}}{\boldsymbol{k}^{2}}\left[\frac{2}{(2 \pi)^{4}} \Phi_{2}(x, \boldsymbol{k},-\boldsymbol{k})\right] .
$$

In $k_{t}$ factorization, the kernel (33) builds up the jet production subprocess in the approximation where two reggeized gluons with momenta $\boldsymbol{k}_{1,2}$ merge into a single gluon with momentum $\boldsymbol{k}_{1}+\boldsymbol{k}_{2}$ which subsequently originates the observed jet:

$$
\begin{gathered}
\frac{d \hat{\sigma}_{g g}\left(x_{1}, \boldsymbol{k}_{1}, x_{2}, \boldsymbol{k}_{2}, y, \boldsymbol{p}\right)}{d y d^{2} \boldsymbol{p}}=\frac{1}{2 s x_{1} x_{2}} \mathcal{K}_{\text {real }}\left(\boldsymbol{k}_{1},-\boldsymbol{k}_{1} ;-\boldsymbol{k}_{2}, \boldsymbol{k}_{2}\right)(2 \pi)^{4} \delta^{(4)}\left(p-k_{1}-k_{2}\right)= \\
=\frac{(2 \pi)^{4}}{s^{2} x_{1} x_{2}} \mathcal{K}_{\text {real }}\left(\boldsymbol{k}_{1},-\boldsymbol{k}_{1} ;-\boldsymbol{k}_{2}, \boldsymbol{k}_{2}\right) \delta^{(2)}\left(\boldsymbol{p}-\boldsymbol{k}_{1}-\boldsymbol{k}_{2}\right) \delta\left(x_{1}-\frac{E_{\perp}\left(\boldsymbol{p}^{2}\right)}{\sqrt{s}} e^{y}\right) \delta\left(x_{2}-\frac{E_{\perp}\left(\boldsymbol{p}^{2}\right)}{\sqrt{s}} e^{-y}\right),
\end{gathered}
$$

where $E_{\perp}\left(\boldsymbol{p}^{2}\right)=\sqrt{m^{2}+\boldsymbol{p}^{2}}$ is the transverse energy of the jet and $m$ its invariant mass (in our case of a single gluon we have $m=0$ ). Using (35), eq. (32) can be cast in the form

$$
\begin{array}{r}
\frac{d \sigma}{d y d^{2} \boldsymbol{p}}(y, \boldsymbol{p})=\frac{s^{2}}{4(2 \pi)^{2}} \int \frac{d^{2} \boldsymbol{k}_{1}}{(2 \pi)^{2}} \int \frac{d^{2} \boldsymbol{k}_{2}}{(2 \pi)^{2}} \int d x_{1} \int d x_{2} \\
\frac{x_{1} \Phi_{2}\left(x_{1} ; \boldsymbol{k}_{1},-\boldsymbol{k}_{1}\right)}{\boldsymbol{k}_{1}^{2} \boldsymbol{k}_{1}^{2}} \frac{d \hat{\sigma}_{g g}}{d y d^{2} \boldsymbol{p}}\left(x_{1}, \boldsymbol{k}_{1}, x_{2}, \boldsymbol{k}_{2}, y, \boldsymbol{p}\right) \frac{x_{2} \Phi_{2}\left(x_{2} ; \boldsymbol{k}_{2},-\boldsymbol{k}_{2}\right)}{\boldsymbol{k}_{2}^{2} \boldsymbol{k}_{2}^{2}}
\end{array}
$$


which, by means of (34), strongly resembles the analogous formula emerging in collinear factorization.

Next we consider the exchange of four reggeized gluons (Fig.15); the corresponding production processes are illustrated in Fig.16. The coupling of the gluons to the proton goes via the functions $\mathcal{N}_{4}$; compared with (36) we restrict ourselves to the limit of small $x_{1}, x_{2}$. We do not write down formulae, but restrict ourselves to a qualitative discussion. Applying
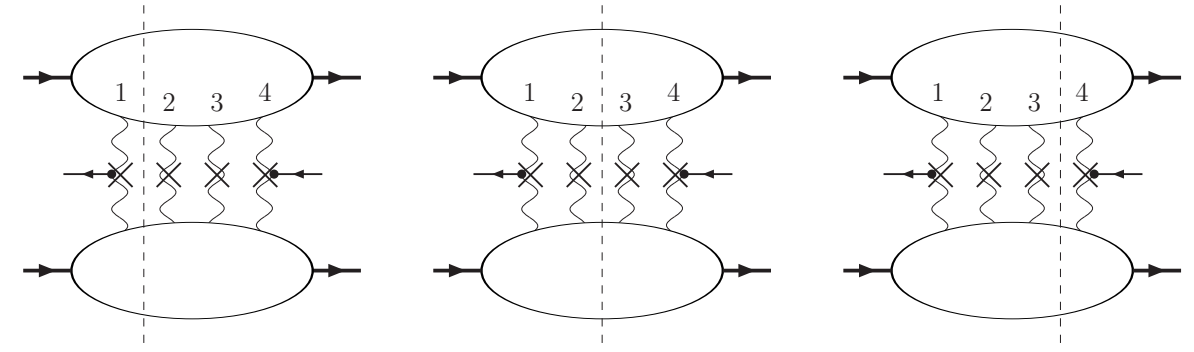

Figure 15: Graphical representation of the process describing the single inclusive cross section.
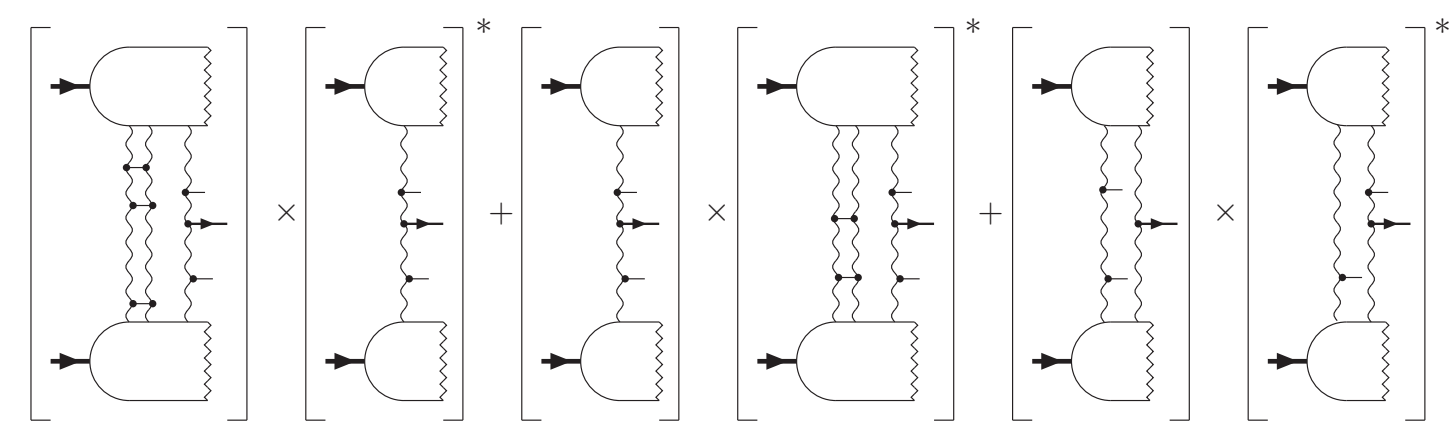

Figure 16: An example of the interference among different processes that produce the cancellation. The line with the arrow in the final state correspond to the emission of the jet.

the counting arguments of the previous section to Fig.15 we will show that all contributions sum up to zero. The symmetry factors $1 / 2$ ! etc. are the same as before; the counting of pairings is slightly different. In the previous case we have asked for multiplicities which has led us to count bound states above and below the $t$-channel intermediate state. Now we compute a one jet inclusive cross section and count the number of possibilities to attach the produced parton (jet). According to our assumptions, the couplings above and below are assumed to be symmetric under the exchange of gluon lines. In the first diagram of Fig.15, the number of ways of attaching the produced parton to the reggeons in the left is 1 , on the right 3 . Therefore, the symmetry factor $-1 / 3$ ! must be multiplied by 3 . The same counting applies to the third diagram of Fig.15. For the diagram in the center we obtain $(1 / 2 !)^{2} \times 2^{2}$ which cancels against the other two diagrams. In summary, the four gluon corrections to the inclusive cross section Fig.14 cancel.

Several remarks have to made about this result. First, all arguments given above apply to the inclusive cross section: in Fig.16 we have illustrated, as an example, a few final states which contribute. These final states alone will not sum up to zero: the cancellations are valid only after summation and integration over all final states partons other than the parton singled out by the jet (marked by an arrow). This means that, for individual events, these AGK cancellations are not visible. It is only after the summation over many events that the 
cancellations work. A necessary ingredient for this are the rescattering contributions (first and second term in Fig.16): if they would be left out, AGK would not work.

Second, the observed jet has introduced the hard scale which is necessary for justifying the use of gluon ladders. Such a hard scale is not present in the other ladders (e.g. in the uncut ladder in the first diagram of Fig.16, or in the cut ladder in the last graph of Fig.16): in the inclusive cross section we sum over final states which might include a large fraction of soft final states. So, strictly speaking, we have demonstrated only the cancellations between hard final states. This is certainly important for the modelling of multiple parton interactions which lead to the production of partons in the final states. However, it is important to include also soft rescattering, i.e. the additional exchange of nonperturbative Pomerons. This can be done by combining the perturbative discussion of this subsection with the nonperturbative one given in subsection 3.1 There we have noted that, when adding soft Pomerons to a single cut Regge pole, the sum over all cuts across the soft Pomerons cancels. If we simply substitute our hard cut Pomeron (containing the produced jet) for this single cut Regge pole, we conclude that, in our pQCD inclusive cross section, also all additional soft Pomeron exchanges cancel. This coincides with the well known result in the collinear factorization which follows from the QCD factorization theorems [13].

A final comment applies to multiple exchanges between the produced jet and one of the hadron projectiles. If one of the two momentum fractions, say $x_{1}$, becomes very small, saturation effects are expected to become important: first corrections of this type are shown in Fig.13. Obviously, they require higher order jet production vertices which have not yet been calculated. These vertices are somewhat analogous to the lowest order coupling of two BFKL ladders to the photon, i.e. to the process: photon + BFKL $\rightarrow$ quark-antiquark. Namely, if we open, in the first figure of Fig.13, the cut Pomeron below the jet vertex, we can view this vertex as the square of the subprocess: gluon + BFKL $\rightarrow$ jet. An important difference between the two cases lies in the fact that the incoming virtual photon is replaced by a colored gluon with transverse momentum $\boldsymbol{k}_{2}$. In this context it is to be expected that the reggeization of the gluon will be an issue: similar to our remarks on $\mathcal{D}_{4}$ (second part of section 2, eq.(5)), there will be pieces which belong to antisymmetric two-gluon states and require a separate discussion. Another question of particular interest is the applicability of the dipole picture: in contrast to the color singlet photon, the incoming gluon carries color and might lead to changes of the impact factor.

We conclude this section with the generalization of our discussion to an arbitrary number of reggeized gluons: in the appendix we show that the cancellation works for a general (even) number of additional reggeized gluons. This leads to the remarkable conclusion that there are no multi-Pomeron corrections to the basic process illustrated in Fig.14: all soft or hard exchanges between the upper and lower projectiles cancel. What remains are only the multiple exchanges between the produced gluon and the upper (or lower) proton.

\subsection{Inclusive double jet production}

We now discuss the inclusive production of two jets, the Mueller-Navelet cross section. The leading contribution is shown in Fig.17a: when the rapidity interval between the two observed jets is large the process is described by Regge kinematics, and the large rapidity interval is due to the BFKL Green function $\mathcal{G}_{2}$, which contains the exchanges of gluons between the reggeized gluons (Fig. 17b); note that, in our convention, $\mathcal{G}_{2}$ does not contain the propagators for the external gluons.

The formula for the cross section associated with Fig.17a is

$$
\frac{x_{1} x_{2} d^{2} \sigma}{d x_{1} d x_{2} d^{2} \boldsymbol{p}_{1} d^{2} \boldsymbol{p}_{2}}\left(x_{1}, x_{2}, y, \boldsymbol{p}_{1}, \boldsymbol{p}_{2}\right)=\frac{1}{4} \int \frac{d^{2} \boldsymbol{k}}{(2 \pi)^{2}} \frac{d^{2} \boldsymbol{k}^{\prime}}{(2 \pi)^{2}}
$$




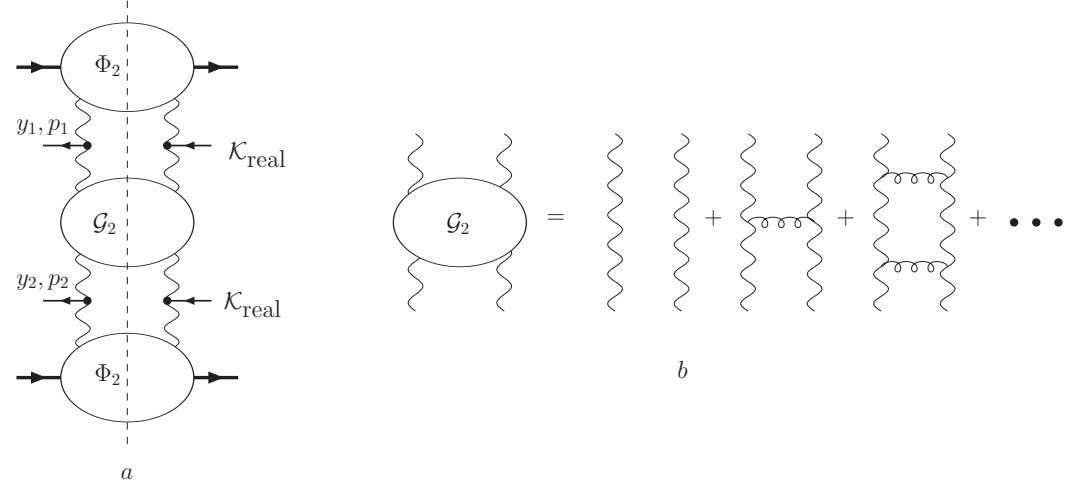

Figure 17: a) Leading term for the inclusive production of two jets with a large rapidity interval between them. b) Definition of the two-to-two reggeized gluons BFKL Green function.

$$
\begin{gathered}
\frac{\Phi_{2}\left(x_{1} ; \boldsymbol{k},-\boldsymbol{k}\right)}{\boldsymbol{k}^{2} \boldsymbol{k}^{2}} \mathcal{K}_{\text {real }}\left(\boldsymbol{k},-\boldsymbol{k} ; \boldsymbol{k}-\boldsymbol{p}_{1},-\boldsymbol{k}+\boldsymbol{p}_{1}\right) \\
\frac{\mathcal{G}_{2}\left(y ; \boldsymbol{k}-\boldsymbol{p}_{1},-\boldsymbol{k}+\boldsymbol{p}_{1} ;-\boldsymbol{k}^{\prime}+\boldsymbol{p}_{2}, \boldsymbol{k}^{\prime}-\boldsymbol{p}_{2}\right)}{\left(\boldsymbol{k}-\boldsymbol{p}_{1}\right)^{2}\left(\boldsymbol{k}-\boldsymbol{p}_{1}\right)^{2}\left(\boldsymbol{k}^{\prime}-\boldsymbol{p}_{2}\right)^{2}\left(\boldsymbol{k}^{\prime}-\boldsymbol{p}_{2}\right)^{2}} \\
\mathcal{K}_{\text {real }}\left(\boldsymbol{k}^{\prime},-\boldsymbol{k}^{\prime} ; \boldsymbol{k}^{\prime}-\boldsymbol{p}_{2},-\boldsymbol{k}^{\prime}+\boldsymbol{p}_{2}\right) \frac{\Phi_{2}\left(x_{2} ; \boldsymbol{k}^{\prime},-\boldsymbol{k}^{\prime}\right)}{\boldsymbol{k}^{\prime 2} \boldsymbol{k}^{\prime 2}} .
\end{gathered}
$$

Defining $\tilde{\mathcal{G}}_{2}$ as the usual Green function but with the propagators for the gluons on the left side of the cut,

$$
\tilde{\mathcal{G}}_{2}\left(y ; \boldsymbol{k}_{1}, \boldsymbol{k}_{2} ; \boldsymbol{k}_{1}^{\prime}, \boldsymbol{k}_{2}^{\prime}\right)=\frac{\mathcal{G}_{2}\left(y ; \boldsymbol{k}_{1}, \boldsymbol{k}_{2} ; \boldsymbol{k}_{1}^{\prime}, \boldsymbol{k}_{2}^{\prime}\right)}{\boldsymbol{k}_{1}^{2} \boldsymbol{k}_{1}^{\prime 2}}
$$

and using (33), (37) can be rewritten as

$$
\begin{gathered}
\frac{x_{1} x_{2} d^{2} \sigma}{d x_{1} d x_{2} d^{2} \boldsymbol{p}_{1} d^{2} \boldsymbol{p}_{2}}\left(x_{1}, x_{2}, y, \boldsymbol{p}_{1}, \boldsymbol{p}_{2}\right)= \\
\frac{1}{4(2 \pi)^{4}} \frac{1}{\boldsymbol{p}_{1}^{2} \boldsymbol{p}_{2}^{2}}\left[\frac{4 \alpha_{s} N_{c}}{(2 \pi)^{2}}\right]^{2} \int d^{2} \boldsymbol{k} \frac{\Phi_{2}\left(x_{1} ; \boldsymbol{k},-\boldsymbol{k}\right)}{\boldsymbol{k}^{2}} \int d^{2} \boldsymbol{k}^{\prime} \frac{\Phi_{2}\left(x_{2} ; \boldsymbol{k}^{\prime},-\boldsymbol{k}^{\prime}\right)}{\boldsymbol{k}^{\prime 2}} \\
\tilde{\mathcal{G}}_{2}\left(y ; \boldsymbol{k}-\boldsymbol{p}_{1},-\boldsymbol{k}+\boldsymbol{p}_{1} ;-\boldsymbol{k}^{\prime}+\boldsymbol{p}_{2}, \boldsymbol{k}^{\prime}-\boldsymbol{p}_{2}\right) .
\end{gathered}
$$

Note that in practice, because of the limited energy of the hadron collider (e.g. the Tevatron), the kinematics of the Mueller-Navelet is chosen such that it maximizes the rapidity gap between the two jets; this implies that the momentum fractions $x_{1}$ and $x_{2}$ are not necessarily small, i.e. the separations in rapidity between the jets and the projectiles $A$ and $B$ are not particularly large. As a result, we have strong $k_{t}$ ordering between the jets and the projectiles, and we are led to the usual integrated gluon densities (eq.(34)). The cross section (37) can be cast in the form

$$
\begin{gathered}
\frac{x_{1} x_{2} d^{2} \sigma}{d x_{1} d x_{2} d^{2} \boldsymbol{p}_{1} d^{2} \boldsymbol{p}_{2}}\left(y, x_{1}, x_{2}, \boldsymbol{p}_{1}, \boldsymbol{p}_{2}\right)= \\
=x_{1} g\left(x_{1}, M^{2}\right)\left[\frac{\alpha_{s} N_{c}}{\boldsymbol{p}_{1}^{2}}\right] \tilde{\mathcal{G}}_{2}\left(y ;-\boldsymbol{p}_{1}, \boldsymbol{p}_{1} ; \boldsymbol{p}_{2},-\boldsymbol{p}_{2}\right)\left[\frac{\alpha_{s} N_{c}}{\boldsymbol{p}_{2}^{2}}\right] x_{2} g\left(x_{2}, M^{2}\right)
\end{gathered}
$$

which is the well known Mueller-Navelet formula for dijet production in hadron-hadron scattering [10]. Also, the incoming gluons with momenta $\boldsymbol{k}_{1}$ and $\boldsymbol{k}_{2}$ may be replaced by quark 
lines, and we have additional contributions from the quark densities. Throughout our discussion of the AGK counting, however, we restrict ourselves to the region of small $x_{1}$ and $x_{2}$.

The first correction to (40) comes from the exchange of four reggeized gluons. Our discussion requires small $x_{1}$ and $x_{2}$, and the coupling of the four gluons proceeds via the function $\mathcal{N}_{4}$. The situation is akin to that shown in Fig.15, but now there are two vertices for the jet emission on each side of the cut. Between the two vertices, the four reggeized gluons interact exchanging gluons; this interaction is symmetric under the exchange of two gluon lines. We denote this interaction by the Green's function $\mathcal{G}_{4}$ (which technically is obtained from the corresponding BKP kernel $[14,15]$ describing the evolution of $n$ interacting reggeized gluon states in the $t$-channel). Following the same line of counting as before, we will find that only a particular subset of the terms gives a non vanishing contribution to the inclusive observable. We treat three different cases, which are illustrated in Fig.18 (where only the

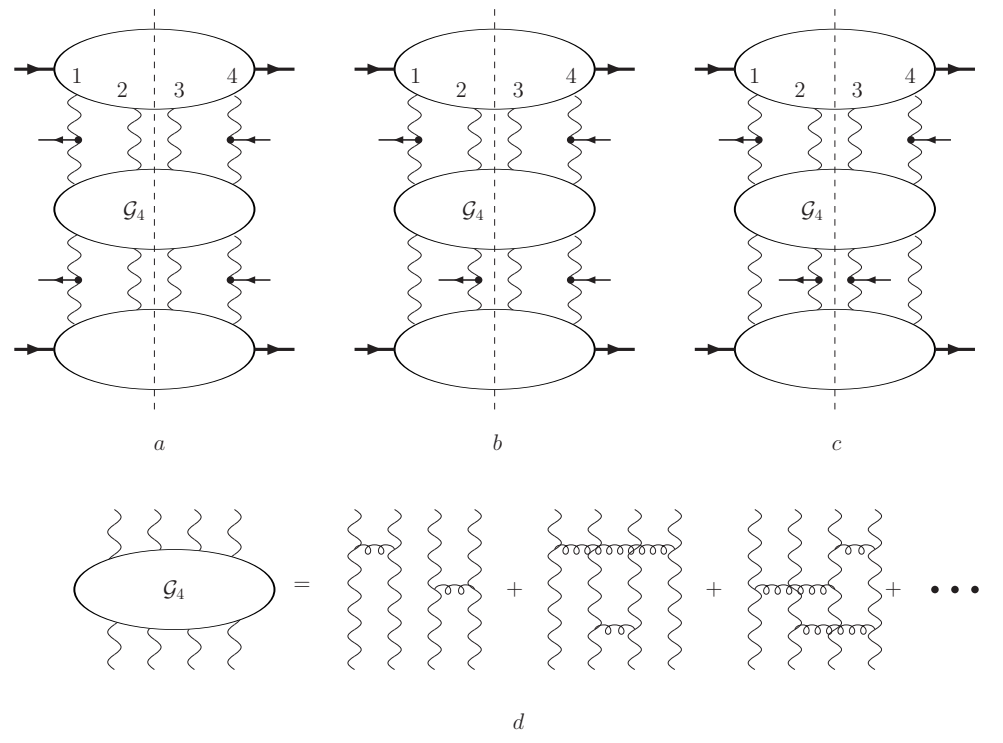

Figure 18: We show the central cut as an example of the classification of the different terms which a priori should contribute to the cross section: a) both vertices attached to the same gluon on both side, b) to the same gluon on one side but to different gluons to the other, and c) to different gluons on both sides. In d) we show the definition of the four-to-four reggeized gluons Green function.

central cut case is shown):

(a) Both production vertices are attached to the same reggeized gluons on each side of the cut; in this case the counting is exactly the same as in the previous section, and the sum of the correction terms vanishes.

(b) Both vertices are attached to the same reggeized gluon on one side of the cut, but to different reggeized gluons on the other side. For example, suppose that the vertices are connected to the same reggeized gluon on the right side (the other case is identical). The first cut (between 1 and 2) does not contribute since there is only one reggeized gluon on the left side. The combinatorial factor for the central cut (between 2 and 3 ) is 2 (the reggeized gluon whom to attach the right sides of the vertices in the right side of the cut can be chosen among two) times 2 (the left side of the first vertex can be attached to one of the two reggeized gluon on the left, but the other vertex can only be attached to the one left); together with the symmetry factor $1 / 4$, the contribution of the diagram is 1 . Finally, the combinatorial factor 
of the third cut (between 3 and 4 ) is $3 \times 2$ because one of the vertices can be attached to one of the three reggeized gluons on the left but the other one to one of the two not connected to the first one. Combining this with the symmetry factor $-1 / 6$, the contribution of the diagram becomes -1 , which cancels exactly the +1 of the central cut.

(c) The vertices are attached to different reggeized gluons on both sides of the cut: only one diagram contributes, and there are no cancellations.

What emerges from this analysis of the four gluon exchange is that those diagrams, in which there are reggeized gluons without a production vertex, give a vanishing contribution. This statement holds for an arbitrary number of reggeized gluons and any number of jets; in particular, diagrams involving the exchange of more that four reggeized gluons do not contribute to the double inclusive jet production. A general proof is given in the Appendix.

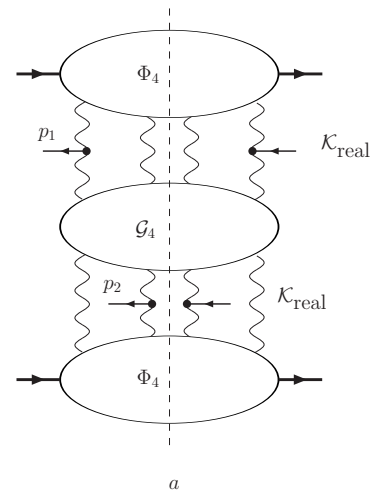

Figure 19: a) Non vanishing correction to the two jets inclusive jet production due to the exchange of four reggeized gluons.

For the case of the Mueller-Navelet jets we are thus left with only one type of corrections (Fig.19): the jets are emitted from different reggeized gluons on both sides of the cut. We write the explicit formula for the correction represented in Fig.19 in the following form:

$$
\begin{gathered}
\frac{x_{1} x_{2} d^{2} \sigma}{d x_{1} d x_{2} d^{2} \boldsymbol{p}_{1} d^{2} \boldsymbol{p}_{2}}\left(y, x_{1}, x_{2}, \boldsymbol{p}_{1}, \boldsymbol{p}_{2}\right)=\frac{1}{4} \int\left[\prod_{i=1}^{4} \frac{d^{2} \boldsymbol{k}_{i}}{(2 \pi)^{2}} \frac{d^{2} \boldsymbol{k}_{i}^{\prime}}{(2 \pi)^{2}}\right](2 \pi)^{2} \delta^{(2)}\left(\sum_{i} \boldsymbol{k}_{i}\right)(2 \pi)^{2} \delta^{(2)}\left(\Sigma_{i} \boldsymbol{k}_{i}^{\prime}\right) \\
\frac{\Phi_{4}\left(x_{1} ; \boldsymbol{k}_{1}, \boldsymbol{k}_{2}, \boldsymbol{k}_{3}, \boldsymbol{k}_{4}\right)}{\boldsymbol{k}_{1}^{2} \boldsymbol{k}_{2}^{2} \boldsymbol{k}_{3}^{2} \boldsymbol{k}_{4}^{2}} \frac{\mathcal{K}_{\text {real }}\left(\boldsymbol{k}_{1}, \boldsymbol{k}_{4} ; \boldsymbol{k}_{1}-\boldsymbol{p}_{1}, \boldsymbol{k}_{4}+\boldsymbol{p}_{1}\right)}{\left(\boldsymbol{k}_{1}-\boldsymbol{p}_{1}\right)^{2}\left(\boldsymbol{k}_{4}+\boldsymbol{p}_{1}\right)^{2}} \\
\mathcal{G}_{4}\left(y ; \begin{array}{c}
\boldsymbol{k}_{1}-\boldsymbol{p}_{1}, \boldsymbol{k}_{2}, \boldsymbol{k}_{3}, \boldsymbol{k}_{4}+\boldsymbol{p}_{1} \\
\boldsymbol{k}_{1}^{\prime}, \boldsymbol{k}_{2}^{\prime}-\boldsymbol{p}_{2}, \boldsymbol{k}_{3}^{\prime}+\boldsymbol{p}_{2}, \boldsymbol{k}_{4}^{\prime}
\end{array}\right) \\
\frac{\mathcal{K}_{\text {real }}\left(\boldsymbol{k}_{2}^{\prime}, \boldsymbol{k}_{3}^{\prime} ; \boldsymbol{k}_{2}^{\prime}-\boldsymbol{p}_{2}, \boldsymbol{k}_{3}^{\prime}+\boldsymbol{p}_{2}\right)}{\left(\boldsymbol{k}_{2}^{\prime}-\boldsymbol{p}_{2}\right)^{2}\left(\boldsymbol{k}_{3}^{\prime}+\boldsymbol{p}_{2}\right)^{2}\left(x_{2} ; \boldsymbol{k}_{1}^{\prime}, \boldsymbol{k}_{2}^{\prime}, \boldsymbol{k}_{3}^{\prime}, \boldsymbol{k}_{4}^{\prime}\right)} \\
\boldsymbol{k}_{1}^{\prime 2} \boldsymbol{k}_{2}^{\prime 2} \boldsymbol{k}_{3}^{\prime 2} \boldsymbol{k}_{4}^{\prime 2}
\end{gathered}
$$

Here we have used another notation for the coupling of the reggeons to the proton, $\Phi_{4}$ rather than $\mathcal{N}_{4}$ : in the limit of small $x_{i}, \Phi_{4}$ coincides with $\mathcal{N}_{4}$. In a more realistic situation we might allow for strong ordering of transverse momenta in those reggeized gluons which connect the jet vertex (cf.our discussion after (39)). As a consequence, the pattern of integrations in (41) is more involved than it was in (39). In particular, the relation between the coupling $\Phi_{4}$ and the vertex functions $\mathcal{N}_{4}$ requires a more detailed discussion.

We finally turn our attention to the Green function $\mathcal{G}_{4}$; since only the central cut survives, there are only diagrams corresponding to multiplicity $k=0,2$. The diffractive case (i.e. the first diagram drawn in Fig.18d) is known in the literature as "hard color singlet" [16]: there 
is a rapidity gap between the two observed jets. Performing the usual counting it is trivially verified that the weight factors of the double multiplicity part of the cut Green function $(k=2)$ is twice the one of the diffractive part $(k=0)$ and, as before, a switch of the pairing structure inside $\mathcal{G}_{4}$ does not spoil this relation.

Specific models $[17,18]$ require the discussion of reggeon diagrams which are more complicated than those discussed in this paper; in particular, this includes diagrams which change the number reggeized gluon in the $t$-channel. For any $t$-channel state with a fixed number of gluons, $2 n$, our discussion applies. However, when considering, for example, double inclusive jet cross sections where between the two emissions the number of gluons changes, one expects to see new contributions where one of the produced jet originates from the gluon number changing vertex. As we have said above, the application of of AGK cutting rules to such vertices requires a separate discussion.

\subsection{Remarks on the relation to phenomenological models}

The topic of multiple interactions has been addressed for many years (see, for example, [3] and references therein, or [9]). Key elements are multi-parton distributions, which are interpreted as probabilities of finding, inside the hadron, a number $n_{p} \geq 1$ of partons with longitudinal momentum fractions $\left\{x_{i}\right\}, i=1, \ldots, n_{p}$. In the framework of $k_{t}$-factorization the partons also carry transverse momenta $\left\{k_{i}\right\}$. These partons then interact through hard subprocesses and produce partonic final states. In the final step, color strings between the produced partons and the remnants of the hadrons describe the hadronization.

The AGK analysis of this paper has mostly been formulated in terms of angular momenta, which are conjugate to energy variables. When translating our results, one first has to emphasize that all the AGK analysis applies to the limit of small momentum fractions $x_{i}$. In particular, in the coupling functions $\mathcal{N}_{n}$ the gluons have longitudinal momentum fractions $x_{i}$ which are all small and defined to be all of the same order. Hence, in our notation we have not specified the dependence upon the $x_{i}$ but rather used the conjugate variable of total angular momentum $\omega$. However, as we have discussed after eq.(4) and illustrated by our example of the eikonal model, it is possible to define more general coupling functions $\mathcal{N}$ by introducing a dependence upon angular momenta of subsystems of gluons, e.g. $\omega_{i j}$. This corresponds to momentum fractions which are small but of different order of magnitude. But, as usual in the small- $x$ approximations, conservation of longitudinal momenta is not observed, and therefore a detailed assignment of longitudinal momenta is not easy. Therefore, as far as the modelling of multiple interactions is concerned, the AGK analysis presented in this paper should mainly be viewed as providing constraints for the limit of small- $x$ values. For a hadron collider which operates at energies as high as the LHC, these constraints should be quite essential.

From the AGK analysis it becomes quite clear that there exist contributions to the cross section which cannot simply be interpreted as probability distributions. Examples have been illustrated in Fig.16: whereas the third contribution represents a square of production amplitudes, and the couplings of the gluons to the upper (or lower) proton might be interpreted as a 'probability of finding two gluons inside a proton', the first and the second contributions are interference terms. Another example of a probabilistic contribution is shown in Fig.19. The third diagram in Fig.20, on the other hand, illustrates an another interference term which survives after all AGK cancellations have been worked out.

One is therefore lead to a more general concept of multiparton correlators. Even in the framework of collinear factorization at the leading twist level, there is already the well-known example of the Generalized Parton Distributions (see [19] for a review), which represent correlations between two partons inside the proton. In this case it is possible to obtain direct experimental information of these correlations by exploiting, for example, the interference 

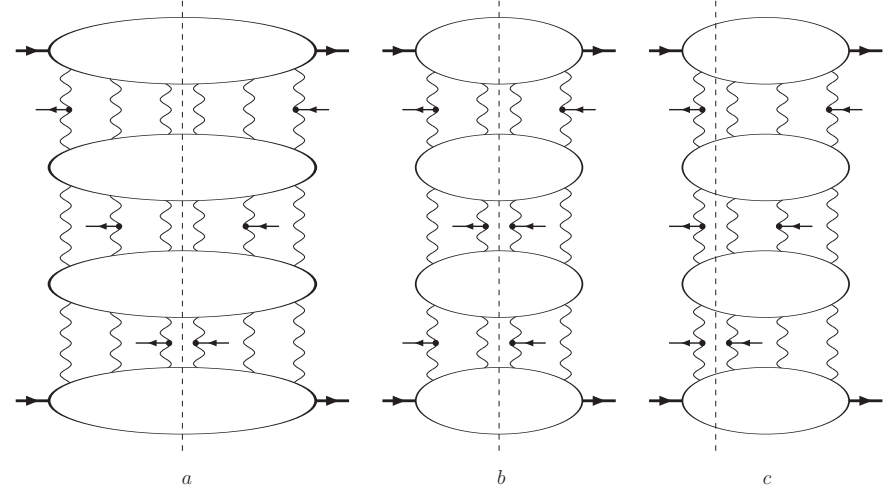

Figure 20: Some diagrams contributing to the three jet production notwithstanding the AGK cancellations.

between the DVCS (Virtual Compton Scattering) and the BH (Bethe-Heitler) process [20]. Beyond these two-parton correlators very little has been worked out in the literature.

\section{Conclusions}

In this paper we have discussed the origin and a few consequences of the AGK cutting rules in pQCD where the Pomeron is described by the BFKL gluon ladders. We have identified the basic ingredient for the validity of the AGK cutting rules, the symmetry properties of couplings of $n$ reggeized gluons to the projectile; they are in agreement with general properties of reggeon unitarity equations, and they have explicitly been verified in pQCD in $\gamma^{*} \gamma^{*}$ (or onium-onium scattering).

As to consequences of the AGK counting rules, we have considered single and double inclusive jet production. In both cases, multipomeron exchanges across the produced jets cancel; this holds for both soft and hard Pomerons. In the two-jet inclusive case, there exists an extra contribution in which the jets are emitted from two different parton chains. As an example, we have analysed how this contribution looks like in in the Mueller-Navelet jet final states; this contribution turns out to be related also to the hard color singlet exchange cross section.

At very small $x$ values, QCD saturation effects are expected to become important. Our AGK analysis leads to the conclusion that such nonlinear corrections should be modelled as multiple exchanges between the jet vertices or between jet vertices and either of the projectiles, but not between the two projectiles.

Several important questions have not been addressed in this paper, and our analysis of AGK counting rules in proton proton scattering remains incomplete. First, QCD allows twogluon states in the $t$-channel that are antisymmetric under momentum and color exchange: in pQCD, bootstrap equations have been shown to be valid, as a result of which the such two-gluon states are identical to a single reggeized gluon. We conjecture that this property holds also beyond pQCD: in this case, as far as the reggeization of gluons is concerned, we do not need to introduce new couplings to the hadron, and our discussion can be reduced to the symmetric functions which ensure the validity of the AGK counting rules. Nevertheless, the existence of these reggeizing pieces require further studies: the most prominent example is $\gamma^{*}$-proton scattering (i.e. the deep inelastic structure function), where in leading order the coupling of four reggeized gluons to the virtual photon consist of reggeized pieces only. This process will be studied in a forthcoming paper. 
In this paper we also have not yet included the Odderon. Perturbative QCD contains an Odderon state, built from (at least) three interacting reggeized gluons. It requires couplings to the proton which satisfy special symmetry properties. Since the intercept of the highest Odderon state known so far [21] lies at unity, at high energies it will be less important; a complete analysis, however, will have to include Odderon exchange.

Another aspect which requires further studies is the limitation due to finite energies. Strictly speaking, the AGK analysis in terms of ladder diagrams requires infinite energies: at any finite energy, the number of produced gluons inside a cut ladder is limited, and, therefore, also the number of different 'cut ladders', $k$, cannot be arbitrarily large. When modelling multiple parton interactions, this may have consequences (e.g. incomplete cancellations) which have to worked out in detail.

\section{Acknowledgements}

M.S. is grateful for the kind hospitality to the II Institut für Theoretische Physik of the Hamburg University where part of this work was done and the Programma Marco Polo for the financial support. G.P.V. is very grateful to the Hamburg University for the warm hospitality. We are grateful to Markus Diehl for his numerous helpful comments. 


\section{Appendix}

In this appendix we present a few details of the generalization of our results to $2 n$ gluons. We start with a few notations. Let $\tilde{\mathcal{A}}^{n}(s, t)$ denote the energy discontinuity of the contribution of $n$-reggeized gluon exchange in the elastic amplitude for the process $A B \rightarrow A B$, $\tilde{\mathcal{F}}^{n}(\omega, \boldsymbol{q})$. The energy discontinuity can be written as the sum over products of production

amplitudes $\tilde{\mathcal{T}}_{A B \rightarrow X}^{j}(s, t)$ for the process $A B \rightarrow X$, where the index $j$ stands for the number of exchanged reggeized gluons. Each such term is of the form $\tilde{\mathcal{T}}_{A B \rightarrow X}^{j}(s, t)\left(\tilde{\mathcal{T}}_{A B \rightarrow X}^{2 n-j}(s, t)\right)^{*}$, i.e. $j$ gluons are exchanged inside the "left" amplitude, $2 n-j$ in the "right" complex conjugate amplitude, and the total number of exchanged gluons is $2 n$. In order to construct $\tilde{\mathcal{A}}^{n}(s, t)$, we have to sum over $j=1, \ldots, 2 n-1$. Furthermore, we also have to sum (denoted by $\sum_{\text {B.S. }}$ ) over all the possibilities to form bound states of two-reggeized gluons bound states (pomerons) inside the amplitudes $\mathcal{N}_{2 n}$ :

$$
\begin{gathered}
\tilde{\mathcal{A}}^{n}(s, t) \stackrel{\text { def }}{=} 2 i \sum_{\text {B.S. }} \sum_{j=1}^{2 n-1} \int d \Omega_{X} \tilde{\mathcal{T}}_{A B \rightarrow X}^{j}\left(s, t_{1}\right)\left(\tilde{\mathcal{T}}_{A B \rightarrow X}^{2 n-j}\left(s, t_{2}\right)\right)^{*}= \\
=2 i \sum_{\text {B.S. }} \sum_{j=1}^{2 n-1} \int d \Omega_{2 n} s^{1+\tilde{\beta}} \mathcal{N}_{2 n} \mathcal{N}_{2 n} \frac{i \xi_{\mathbb{G}}\left(\boldsymbol{k}_{1}\right) \ldots i \xi_{\mathbb{G}}\left(\boldsymbol{k}_{j}\right)}{j !} \frac{\left[i \xi_{\mathbb{G}}\left(\boldsymbol{k}_{j+1}\right) \ldots i \xi_{\mathbb{G}}\left(\boldsymbol{k}_{2 n}\right)\right]^{*}}{(2 n-j) !}
\end{gathered}
$$

Here we have made used of the fact that $\mathcal{N}_{2 n}$ does not depend on the position of the cut. The tilde symbol indicates that we are working in pQCD. Since the signature factor $\xi_{\mathbb{G}}(\boldsymbol{k})=$ $-2 / \pi \boldsymbol{k}^{2}$ is real (cf. the discussion after eq.(13)), (42) can be cast into the form

$$
\tilde{\mathcal{A}}^{n}(s, t)=2^{n} \pi i \sum_{\text {B.S. }} \sum_{j=1}^{2 n-1} S_{j}^{n} \int d \Omega_{2 n} s^{1+\tilde{\beta}_{n}} \mathcal{N}_{2 n} \mathcal{N}_{2 n} \tilde{\gamma}_{\left\{\boldsymbol{k}_{l}\right\}}
$$

where we have used (16), and we have introduced the symmetry factor

$$
S_{j}^{n}=\frac{(-1)^{n-j}}{j !(2 n-j) !}
$$

\section{Inclusive case}

We begin with the fully inclusive case of section 3. Starting from (42) we want to find a decomposition in terms of cut Pomerons. For each position of the cut (denoted by $j$ ) we first classify all the different possibilities of forming 2-gluon bound states out of the $2 n$ reggeized gluons; at the same time we have to keep track of how many of these bound states are cut by the energy cutting line. Let $k$ denote the number of such cut bound states: then $k$ also labels the multiplicity of $s$-channel gluons intersected by the cutting lines. We then must count how many different configurations $\left(i_{1} j_{1}\right) \ldots\left(i_{n} j_{n}\right)$ contribute to a term belonging to the multiplicity $k$.

Denoting by $C^{n}$ the number of different pairings among $2 n$ reggeized gluons, is clear that $C^{n}=(2 n-1) !$ !: starting with the first reggeized gluon to the left of the cutting line, its partner can be chosen among $2 n-1$ other $t$-channel gluons; the partner of the next unpaired reggeized gluon can be chosen among $2 n-3$ other gluons, and so forth until all the reggeized gluons have been put into pairs. Next we will decompose $C^{n}$ into contributions with fixed-multiplicity $k$ :

$$
C^{n}=\sum_{k} C_{j k}^{n},
$$


where $C_{j k}^{n}$ is the number of configurations that contain exactly $k$ cut pairs. The subscript $j$ indicates that this number will depend upon the position of the cutting line. It is clear that $C_{j k}^{n}$ is 0 unless $k$ and $j$ are both even or both odd, i.e. $C_{j k}^{n} \propto 1 / 2\left(1+(-1)^{j+k}\right)$. Also, we need $k \leq j$ and $k \leq 2 n-j$, i.e. the range of $k$ values in 45 depends upon $j$. If $j$ and $k$ satisfy these conditions we can chose $k$ reggeized gluons in each side of the cut in $\left(\begin{array}{l}j \\ k\end{array}\right)\left(\begin{array}{c}2 n-j \\ k\end{array}\right)$ different ways and couple each reggeized gluon in the left side with a reggeized gluon in the right side (this can be done in $k$ ! ways). We are left with $j-k$ reggeized gluons on the left side and $2 n-j-k$ on the right side, which must be coupled in pairs without crossing the cut; there are $(j-k-1) ! !(2 n-j-k-1)$ !! ways to do that. Eventually the expression obtained is

$$
C_{j k}^{n}=\frac{1+(-1)^{j+k}}{2} \frac{j !(2 n-j) !}{k !(j-k) ! !(2 n-j-k) ! !},
$$

and it is easy to verify that (46) satisfies (45).

With this expression for $C_{j k}^{n}$ we can rewrite (42) as a sum over the multiplicity index $k$ of fixed-multipicity contributions $\tilde{\mathcal{A}}_{k}^{n}(s, t)$ :

$$
\tilde{\mathcal{A}}^{n}(s, t)=\sum_{k=0}^{n} \tilde{\mathcal{A}}_{k}^{n}(s, t)
$$

where

$$
\tilde{\mathcal{A}}_{k}^{n}(s, t)=2^{n} \pi i \sum_{j=k}^{2 n-k} S_{j}^{n} C_{j k}^{n} \int d \Omega_{2 n} s^{1+\tilde{\beta}} \mathcal{N}_{2 n} \mathcal{N}_{2 n} \tilde{\gamma}_{\left\{\boldsymbol{k}_{l}\right\}}
$$

The reader should note that the integral in (48) does not depend upon $j$ or $k$; therefore there is just a combinatorial factor in front of the integral. Performing the sum in (48) we arrive at:

$$
\begin{aligned}
\sum_{j=k}^{2 n-k} S_{j}^{n} C_{j k}^{n} & =\frac{(-1)^{n}}{2 k !} \sum_{j=k}^{2 n-k} \frac{(-1)^{j}+(-1)^{k}}{(j-k) ! !(2 n-j-k) ! !} \\
& = \begin{cases}\frac{(-1)^{n}}{n !}\left(1-2^{1-n}\right) & \text { if } k=0 \\
\frac{(-1)^{n-k}}{k !(n-k) !} & \text { if } k>0\end{cases}
\end{aligned}
$$

With the definition (15), we have shown that (48) can be written as in (14).

\section{Switches of Pairings}

Now we want to show that a switch from a configuration $\left(i_{1} j_{1}\right) \ldots\left(i_{n} j_{n}\right)$ to $\left(i_{1}^{\prime} j_{1}^{\prime}\right) \ldots\left(i_{n}^{\prime} j_{n}^{\prime}\right)$ preserves the relative weight between different multiplicities $k$. The situation is the following: at some point in rapidity we have the configuration $\left(i_{1} j_{1}\right) \ldots\left(i_{n} j_{n}\right)$ with muliplicity $k$, and the weight factor of this multiplicity $k$ is the usual $\tilde{\mathcal{F}}_{k}^{n}$ given in (15); moving now to a neighbouring rapidity interval we switch to a different configuration $\left(i_{1}^{\prime} j_{1}^{\prime}\right) \ldots\left(i_{n}^{\prime} j_{n}^{\prime}\right)$, and we want to compute the weight of the term with $k^{\prime}$ cut rungs. Contributions to this $k^{\prime}$ can come from different $k$ terms before the switch; we therefore must sum over $k$ the $\tilde{\mathcal{F}}_{k}^{n}$ muliplied by the number of ways of obtaining a configuration with $k^{\prime}$ cut rungs (normalized by the total number).

Put in other words, we must build an $(n+1) \times(n+1)$ matrix, where the initial multiplicity $k$ labels the columns, and the final multiplicity $k^{\prime}$ labels the rows; the elements $\mathcal{M}_{k^{\prime} k}^{n}$ of this matrix are defined to be the fraction of configurations which, after the switch, lead to $k^{\prime}$ cut 
rungs. We then want to show that the vector $\tilde{\mathcal{F}}_{k}^{n}$ is an eigenvector of this matrix $\mathcal{M}_{k^{\prime} k}^{n}$. Using a vector notation and dropping the index $n$ we want to show that:

$$
\mathcal{F} \propto \mathcal{M} \mathcal{F}
$$

But $\mathcal{F}$ is an eigenvector of $\mathcal{M}$ iff it is an eigenvector of $\mathcal{M}+c \mathbb{1}$ where $c$ is an arbitrary constant. Note that, by definition, $\mathcal{M}$ is computed by considering only transitions between different pairwise configurations, and a transition to the same configuration, in our matrix notation, is proportional to the identity (the nature of the cut does not change). The proportionality constant is just $1 / C^{n}$, where $C^{n}$ is the total number of configurations defined before. We normalize the matrix $\mathcal{M}$ by dividing by $C^{n}-1$ instead of $C^{n}$, because we are considering only transitions to different configurations; of course, this normalization does not affect the form of the eigenvectors (it changes only the eigenvalues). So we can change its normalization multiplying by $\left(C^{n}-1\right) / C^{n}$. Eventually, proving (50) is equivalent to prove

$$
\mathcal{F} \propto \overline{\mathcal{M}} \mathcal{F}
$$

where we have introduced the new matrix $\overline{\mathcal{M}}$ defined by

$$
\overline{\mathcal{M}}=\frac{\left(C^{n}-1\right) \mathcal{M}+\mathbb{1}}{C^{n}} .
$$

This new matrix $\overline{\mathcal{M}}$ now contains all possible switches to a new configuration, not only to the different ones.

Instead of computing explicitely the coefficients of $\overline{\mathcal{M}}$, it is easier to compute directly the RHS of (51), i.e. write the sum over the various contributions due to different positions of the cut before the switch. For fixed $j$ and $k$ it is trivial to obtain the fraction of configurations with $k^{\prime}$ cut rungs: it is $C_{j k^{\prime}}^{n} / C^{n}$ (note that this is true only because we are no longer restricting ourselves to the new configuration being different from the previous one). Therefore, using

$$
\tilde{\mathcal{F}}_{k}^{n}=2^{n-1} n ! \sum_{j=k}^{2 n-k} S_{j}^{n} C_{j k}^{n}
$$

the rhs of (51) can be written as

$$
\sum_{k=0}^{n} 2^{n-1} n ! \sum_{j=k}^{2 n-k} \frac{C_{j k^{\prime}}^{n}}{C^{n}} S_{j}^{n} C_{j k}^{n}
$$

After using (45), this gives exactly $\tilde{\mathcal{F}}_{k^{\prime}}^{n}$, i.e. the $\tilde{\mathcal{F}}_{k}^{n}$ form an eigenvector.

\section{1-Jet inclusive}

In this subsection we generalize the cancellation of diagrams with $n>2$ gluon lines in the single inclusive cross section. The discontinuity of the amplitude for single jet production is given by

$$
\tilde{\mathcal{A}}_{1 \text {-jet }}^{n}(s, t)=2 i \sum_{\text {B.S. }} \sum_{j=1}^{2 n-1} j(2 n-j) S_{j}^{n} \int d \Omega_{2 n} s^{1+\tilde{\beta}} \mathcal{N}_{2 n} \mathcal{N}_{2 n} \tilde{\gamma}_{\left\{\boldsymbol{k}_{l}\right\}},
$$

where the factor $j(2 n-j)$ counts the number of ways in which the jet can be connected to the reggeized gluons on the lhs and rhs of the cutting line. Since now we are not interested in 
counting the number of cut pomerons, the sum over different bound state configuration gives just a global factor $C^{n}$. Performing the summation over the position of the cut $j$ we get

$$
\begin{aligned}
\sum_{j=1}^{2 n-1} j(2 n-j) S_{j}^{n} & =(-1)^{n} \sum_{1}^{2 n-1} \frac{(-1)^{j}}{(j-1) !(2 n-j-1) !} \\
& =(-1)^{n-1} \sum_{0}^{2 n-2} \frac{(-1)^{j}}{j !(2 n-j-2) !} \\
& =\left\{\begin{array}{ll}
1 & \text { if } n=1 \\
0 & \text { if } n>1
\end{array},\right.
\end{aligned}
$$

and we have the result

$$
\tilde{\mathcal{A}}_{1-\mathrm{jet}}^{n}(s, t)=0 \quad \text { if } n>1 \text {. }
$$

\section{2-Jet inclusive}

The situation is similar to the previous one: the combinatoric factor $j(2 n-j)$ is replaced by $j^{2}(2 n-j)^{2}$. The separation in the three cases analyzed in section 4.2 can formally be obtained by writing one of the factors $j$ as $(j-1)+1$ and one of the factors $2 n-j$ as $(2 n-j-1)+1$; the combinatorial factor $j^{2}(2 n-j)^{2}$ is therefore written as a sum of four terms:

$$
j^{2}(2 n-j)^{2}=j(j-1)(2 n-j)(2 n-j-1)+j(2 n-j)(2 n-j-1)+j(j-1)(2 n-j)+j(2 n-j) .
$$

Let us also classify these terms counting the numbers $\left(n_{l}, n_{r}\right)$ of reggeized gluons on the (left, right) side of the cut which emit at least one gluon (from the jet vertex). If $n=1$ clearly only the last term in eq. (58) is present (since $j=1$ ) and this correspond to the case $\left(n_{l}, n_{r}\right)=(1,1)$. For $n \geq 2$ all the terms are not trivial:

- $j(j-1)(2 n-j)(2 n-j-1)$, corresponding to the jets being connected to different reggeized gluons on both sides, $\left(n_{l}, n_{r}\right)=(2,2)$;

- $j(2 n-j)(2 n-j-1)$, corresponding to the jets being connected to different reggeized gluons on the right hand side but to the same gluon on the left hand side, $\left(n_{l}, n_{r}\right)=$ $(1,2)$;

- $j(j-1)(2 n-j)$, corresponding to the jets being connected to different reggeized gluons on the left hand side but to the same gluon on the right hand side, $\left(n_{l}, n_{r}\right)=(2,1)$;

- $j(2 n-j)$, corresponding to the jets being connected to the same reggeized gluons on both sides, $\left(n_{l}, n_{r}\right)=(1,1)$.

It is easy to verify that after multiplication with the usual symmetry factor and summation over $j$, the last three term vanishes independently and the first one vanishes unless $n=2$, so that the only surving contributions appear when $n=\left(n_{l}+n_{r}\right) / 2$.

\section{$m$-jet inclusive}

We are now ready to generalize this result to the emission of $m$ jets. The contribution of the $2 n$-reggeized gluon diagram is

$$
\tilde{\mathcal{A}}_{m \text {-jet }}^{n}(s, t)=2 i \sum_{\text {B.S. }} \sum_{j=1}^{2 n-1} S_{j}^{n} j^{m}(2 n-j)^{m} \int d \Omega_{2 n} s^{1+\tilde{\beta}} \mathcal{N}_{2 n} \mathcal{N}_{2 n} \tilde{\gamma}_{\left\{\boldsymbol{k}_{l}\right\}} .
$$


With the manipulations described in the previous subsection, we can reduce this expression to a sum of terms, each of which corresponds to a diagram in which a certain number $n_{r}$ of reggeized gluons on the right side of the cut emit at least one gluon, and $n_{l}$ reggeized gluons on the left hand side emit one or more gluons. Each term is of the form

$$
\begin{aligned}
(-1)^{n} \sum_{j=n_{l}}^{2 n-n_{r}} \frac{(-1)^{j}}{j !(2 n-j) !} & j(j-1) \ldots\left(j-n_{l}+1\right) \cdot(2 n-j)(2 n-j-1) \ldots\left(2 n-j-n_{r}+1\right) \\
& =\quad(-1)^{n} \sum_{n_{l}}^{2 n-n_{r}} \frac{(-1)^{j}}{\left(j-n_{l}\right) !\left(2 n-j-n_{r}\right) !} \\
& =\quad(-1)^{n} \sum_{0}^{2 n-n_{r}-n_{l}} \frac{(-1)^{j+n_{l}}}{j !\left(2 n-j-n_{r}-n_{l}\right) !} \\
& =\frac{(-1)^{n_{l}+n_{r}}}{\left(2 n-n_{l}-n_{r}\right) !} \sum_{0}^{2 n-n_{r}-n_{l}\left(\begin{array}{c}
2 n-n_{l}-n_{r} \\
j
\end{array}\right)(-1)^{j}} \\
& = \begin{cases}(-1)^{\frac{n_{l}-n_{r}}{2}} & \text { if } n=\frac{n_{l}+n_{r}}{2} \\
0 & \text { if } n>\frac{n_{l}+n_{r}}{2}\end{cases}
\end{aligned}
$$

Since $n_{l, r} \leq m$, the inequality $\left(n_{l}+n_{r}\right) / 2 \leq m$ holds, and all diagrams with $n>m$ vanish. Among the others, the non-vanishing ones are those which satisfy the condition $n=\left(n_{l}+\right.$ $\left.n_{r}\right) / 2$. Briefly the condition is

$$
2 n=n_{l}+n_{r}, \quad m>n
$$

and it fixes also the position of the cut to $j=n_{l}$.

The vanishing of (59) for $n>m$ can also be seen in another way:

$$
\begin{aligned}
\sum_{j=1}^{2 n-1} S_{j}^{n} j^{m}(2 n-j)^{m} & =\sum_{1}^{2 n-1} \frac{(-1)^{n-j}}{j !(2 n-j) !} j^{m}(2 n-j)^{m} \\
& =\frac{1}{(2 n) !} \sum_{0}^{2 n}\left(\begin{array}{c}
2 n \\
j
\end{array}\right)(-1)^{n-j} j^{m}(2 n-j)^{m} \\
& =\left.\frac{(-1)^{n}}{(2 n) !} \frac{\partial^{2 m}}{\partial \alpha^{m} \partial \beta^{m}} e^{2 n \beta} \underbrace{\sum_{0}^{2 n}\left(\begin{array}{c}
2 n \\
j
\end{array}\right)(-1)^{2 n-j} e^{(\alpha-\beta) j}}_{=\left(e^{\alpha-\beta}-1\right)^{2 n}}\right|_{\alpha, \beta=0} \\
& =\left.\frac{(-1)^{n}}{(2 n) !} \frac{\partial^{2 m}}{\partial \alpha^{m} \partial \beta^{m}}\left(e^{\alpha}-e^{\beta}\right)^{2 n}\right|_{\alpha, \beta=0} \\
& =\left.\frac{(-1)^{n}}{(2 n) !}\left(x \frac{\partial}{\partial x}\right)^{m}\left(y \frac{\partial}{\partial y}\right)^{m}(x-y)^{2 n}\right|_{x, y=1}
\end{aligned}
$$

The operator $\left(x \partial_{x}\right)^{m}$ can be written as

$$
\left(x \frac{\partial}{\partial x}\right)^{m}=\sum_{k=1}^{m} a_{k}^{m} x^{k} \frac{\partial^{k}}{\partial x^{k}},
$$

where the coefficient $a_{k}^{m}$ are positive integer numbers whose explicit expression is not needed. Using (63) in (62) we obtain

$$
\begin{array}{r}
\left.\frac{(-1)^{n}}{(2 n) !} \sum_{k, k^{\prime}=1}^{m} a_{k}^{m} a_{k^{\prime}}^{m} x^{k} y^{k^{\prime}} \frac{\partial^{k}}{\partial x^{k}} \frac{\partial^{k^{\prime}}}{\partial y^{k^{\prime}}}(x-y)^{2 n}\right|_{x, y=1}= \\
(-1)^{n} \sum_{k, k^{\prime}=1}^{m} \delta_{k+k^{\prime}, 2 n} a_{k}^{m} a_{k^{\prime}}^{m}(-1)^{k^{\prime}}
\end{array}
$$


which vanishes if $n>m$.

\section{References}

[1] V. A. Abramovsky, V. N. Gribov and O. V. Kancheli, Yad. Fiz. 18 (1973) 595 [Sov. J. Nucl. Phys. 18 (1974) 308].

[2] L. N. Lipatov, Sov. J. Nucl. Phys. 23 (1976) 338 [Yad. Fiz. 23 (1976) 642].

E. A. Kuraev, L. N. Lipatov and V. S. Fadin, Sov. Phys. JETP 45 (1977) 199 [Zh. Eksp. Teor. Fiz. 72 (1977) 377].

I. I. Balitsky and L. N. Lipatov, Sov. J. Nucl. Phys. 28 (1978) 822 [Yad. Fiz. 28 (1978) 1597].

[3] T. Sjostrand and P. Z. Skands, JHEP 0403 (2004) 053 [arXiv:hep-ph/0402078]. Eur.Phys.J.C39:129-154,2005 e-Print Archive: hep-ph/0408302

[4] M. A. Braun, Phys. Lett. B 483 (2000) 105 [arXiv:hep-ph/0003003].

[5] Y. V. Kovchegov and E. Levin, Nucl. Phys. B 577 (2000) 221 [arXiv:hep-ph/9911523].

[6] J. Bartels and M. G. Ryskin, Z. Phys. C 76 (1997) 241 [arXiv:hep-ph/9612226].

[7] J. Bartels and M. Wusthoff, Z. Phys. C 66 (1995) 157.

[8] J. Bartels and C. Ewerz, JHEP 9909 (1999) 026 [arXiv:hep-ph/9908454].

[9] R. Ragazzon and D. Treleani, Phys. Rev. D 53 (1996) 55 [arXiv:hep-ph/9508286].

[10] A. H. Mueller and H. Navelet, Nucl. Phys. B 282 (1987) 727.

[11] H. D. I. Abarbanel, J. B. Bronzan, R. L. Sugar and A. R. White, Phys. Rept. 21 (1975) 119.

[12] V. N. Gribov, I. Y. Pomeranchuk and K. A. Ter-Martirosian, Phys. Rev. 139 (1965) B184.

V. N. Gribov, I. Y. Pomeranchuk and K. A. Ter-Martirosian, Sov. J. Nucl. Phys. 2 (1965) 258 [Yad. Fiz. 2 (1965) 361].

[13] J. C. Collins, D. E. Soper and G. Sterman, Adv. Ser. Direct. High Energy Phys. 5 (1988) 1 [arXiv:hep-ph/0409313].

[14] J. Bartels, Nucl. Phys. B 151 (1979) 293.

J. Bartels, Nucl. Phys. B 175 (1980) 365.

J. Kwiecinski and M. Praszalowicz, Phys. Lett. B 94 (1980) 413.

[15] T. Jaroszewicz, Acta Phys. Polon. B 11 (1980) 965.

[16] A. H. Mueller and W. K. Tang, Phys. Lett. B 284 (1992) 123.

B. Cox, J. Forshaw and L. Lonnblad, JHEP 9910 (1999) 023 [arXiv:hep-ph/9908464]. R. Enberg, G. Ingelman and L. Motyka, Phys. Lett. B 524 (2002) 273 [arXiv:hepph/0111090].

[17] Y. V. Kovchegov and K. Tuchin, Phys. Rev. D 65 (2002) 074026 [arXiv:hep-ph/0111362]; J. Jalilian-Marian and Y. V. Kovchegov, Phys. Rev. D 70 (2004) 114017 [arXiv:hepph/0405266]. 
[18] M. A. Braun, arXiv:hep-ph/0502184.

[19] M. Diehl, Phys. Rept. 388 (2003) 41 [arXiv:hep-ph/0307382].

[20] M. Diehl, T. Gousset, B. Pire and J. P. Ralston, Phys. Lett. B 411 (1997) 193 [arXiv:hepph/9706344].

A. V. Belitsky, D. Muller, L. Niedermeier and A. Schafer, Nucl. Phys. B 593 (2001) 289 [arXiv:hep-ph/0004059].

A. V. Belitsky, D. Muller and A. Kirchner, Nucl. Phys. B 629 (2002) 323 [arXiv:hep$\mathrm{ph} / 0112108]$.

[21] J. Bartels, L. N. Lipatov and G. P. Vacca, Phys. Lett. B 477 (2000) 178 [arXiv:hep$\mathrm{ph} / 9912423]$. 